\title{
Physics-guided machine learning approaches to predict the ideal stability properties of fusion plasmas
}

To cite this article before publication: Andrea Piccione et al 2020 Nucl. Fusion in press https://doi.org/10.1088/1741-4326/ab7597

\section{Manuscript version: Accepted Manuscript}

Accepted Manuscript is "the version of the article accepted for publication including all changes made as a result of the peer review process, and which may also include the addition to the article by IOP Publishing of a header, an article ID, a cover sheet and/or an 'Accepted

Manuscript' watermark, but excluding any other editing, typesetting or other changes made by IOP Publishing and/or its licensors"

This Accepted Manuscript is @ 2020 IAEA, Vienna.

During the embargo period (the 12 month period from the publication of the Version of Record of this article), the Accepted Manuscript is fully protected by copyright and cannot be reused or reposted elsewhere.

As the Version of Record of this article is going to be / has been published on a subscription basis, this Accepted Manuscript is available for reuse under a CC BY-NC-ND 3.0 licence after the 12 month embargo period.

After the embargo period, everyone is permitted to use copy and redistribute this article for non-commercial purposes only, provided that they adhere to all the terms of the licence https://creativecommons.org/licences/by-nc-nd/3.0

Although reasonable endeavours have been taken to obtain all necessary permissions from third parties to include their copyrighted content within this article, their full citation and copyright line may not be present in this Accepted Manuscript version. Before using any content from this article, please refer to the Version of Record on IOPscience once published for full citation and copyright details, as permissions will likely be required. All third party content is fully copyright protected, unless specifically stated otherwise in the figure caption in the Version of Record.

View the article online for updates and enhancements. 


\title{
Physics-guided machine learning approaches to predict the ideal stability properties of fusion plasmas
}

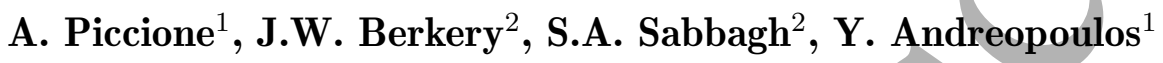 \\ ${ }^{1}$ Department of Electronic and Electrical Engineering, University College London, \\ WC1E 7JE, UK \\ ${ }^{2}$ Department of Applied Physics and Applied Mathematics, Columbia University, \\ New York, NY 10027, USA \\ E-mail: a.piccione@ucl.ac.uk
}

\begin{abstract}
One of the biggest challenges to achieve the goal of producing fusion energy in tokamak devices is the necessity of avoiding disruptions of the plasma current due to instabilities. The Disruption Event Characterization and Forecasting (DECAF) framework has been developed in this purpose, integrating physics models of many causal events that can lead to a disfuption. Two different machine learning approaches are proposed to improve the ideal magnetohydrodynamic (MHD) no-wall limit component of the kinetic stability model included in DECAF. First, a random forest regressor (RFR), was adopted to reproduce the DCON computed change in plasma potential energy without wall effects, $\delta W_{n o-w a l l}^{n=1}$, for a large database of equilibria from the National Spherical Torus Experiment (NSTX). This tree-based method provides an analysis of the contribution of each input feature, giving an insight into the underlying physics phenomena. Secondly, a fully-connected neural network has been trained on sets of calculations with the DCON code, to get an improved closed form equation of the no-wall $\beta$ limit as a function of the relevant plasma parameters indicated by the RFR. The neural network has been guided by physics theory of ideal MHD in its extension outside the domain of the NSTX experimental data. The estimated value of $\beta_{N, \text { no-wall }}^{n=1}$ has been incorporated into the DECAF kinetic stability model and tested against a set of experimentally stable and unstable discharges. Moreover, the neural network results were used to simulate a real-time stability assessment using only quantities available in real-time. Finally, the portability of the model was investigated, showing encouraging results by testing the NSTX-trained algorithm on the Mega Ampere Spherical Tokamak (MAST).
\end{abstract}

\section{Introduction}

High temperature plasmas being studied in tokamak magnetic confinement devices for the purposes of fusion energy must be maintained at high pressures in order to achieve good performance. Unfortunately, at high ratios of plasma pressure to magnetic confinement field pressure, known as $\beta$, these plasmas are subject to magnetohydrodynamic (MHD) instabilities that can lead to disruption of the plasma current and impact of the plasma energy on the walls of the device. In order to study 


\section{Physics-guided ML approaches for ideal stability forecasting}

and prevent these disruptions, the Disruption Event Characterization and Forecasting (DECAF) code $[1,2,3]$ has been constructed. This code incorporates many different physics models for various causes of disruptions. One of these is the kinetic stability module [1], which directly addresses the issue of high plasma $\beta$ leading to modes of instability called resistive wall modes (RWMs) and accounts for the stabilizing effects of plasma particle motions $[4,5,6]$. Included in this kinetic module is the ideal stability model, which does not account for drift kinetic model effects, but provides the basis for understanding when the plasma has reached a dangerous state and computes quantities that are directly input into the larger kinetic model [7]. The ultimate goal of the DECAF approach is to provide real-time forecasting of plasma stability. Since the computations of both ideal and kinetic stability by traditional means, such as by the DCON [8] and MISK [9] codes, respectively, are too computationally time consuming to be performed in real-time, alternative approaches are necessary. For example, efforts have been made to speed up the DCON ideal stability calculation by orders of magnitude [10]. This approach has advantages, as a full MHD calculation can give the stability of any equilibrium solution to the Grad-Shafranov equation, uniquely specified by the plasma boundary shape, as well as pressure and safety factor profiles. In fact, a neural network could also be trained on a large set of stability calculations of model equilibria with these inputs in order to emulate real-time calculations as well. A different approach has been used previously with success: to formulate analytic reduced models for both the ideal [7] and kinetic [1] stability, which maintain the physics basis of the more complex code calculations. In this paper, an extension of that idea is explored, with the help of machine learning (ML).

An important new direction in ML applications is to employ physics-guided, or hybrid, techniques. That is to say, rather than simply feeding raw experimental data into a ML algorithm, physics knowledge of the problem should be utilized to pre-process input into the machine learning tools, as well as to interpret the output. On the other hand, the patterns discovered through the computations can also be used to improve the physics knowledge, by suggesting previously unappreciated dependencies, for example. In this paper we make the first steps in the direction of this hybrid approach for the specific problem at hand ideal stability analysis of tokamak plasmas.

Machine learning approaches continue to show outstanding performance in classification tasks. Early-stage neural networks have been proposed as a method for disruption prediction many years ago $[11,12,13,14,15,16,17]$ and interest has recently grown rapidly $[18,19,20]$ with the advent of modern neural network designs that use appropriate non-linearities (i.e. parametric ReLU), customized cost functions for classification problems, and training methods that converge to optima that are shown to be sufficiently stable and surpass conventional optimization methods, like those based on convex approximations. Additionally, neural networks are now being used to model the outputs of plasma physics codes, for example in the area of particle transport [21, 22] or neutral beam injection [23]. Here we use two different algorithms to classify the output of DCON into stable and unstable regions, for a set of calculations 


\section{Physics-guided ML approaches for ideal stability forecasting}

for the NSTX tokamak. A large database of equilibria from NSTX has been analyzed with DCON, providing the ideal change in plasma potential energy due to a perturbation of the confining magnetic field without the presence of a conducting wall, $-\delta W_{n o-w a l t}^{n=1}$. $\delta W_{n o-w a l l}^{n=1}$ changes from positive to negative when the plasma changes from ideal stable to unstable. We use the negative of the change in potential energy throughout the present paper so that, more intuitively, negative values are below the limit (stable) and positive quantities are above (unstable). For determining the ideal, and ultimately kinetic mode growth rates, the value of $\beta_{N, n o-w a l l}^{n=1}$ and both the value and the sign of $-\delta W_{n o-w a l l}^{n=1}$ must be determined. To that end, we have initially developed a machine learning based approach to determine $-\delta W_{n o-\text { wall }}^{n=1}$ as a function of several plasma parameters. We have tested with both multilayer perceptron (MLP) artificial neural networks and random forest regression (RFR)[24] and we found that the RFR outperformed MLPs in terms of R-squared and flexibility. This model provides an emulation of DCON that could actually run in real-time. The random forest technique has also been recently employed in plasma physics research, specifically in disruption warning, prediction and survival analysis, where a real-time random forest based predictor was employed on the DIII-D, Alcator C-Mod and EAST tokamaks [25, 26, 27, 28, 29]. However, no such approach has been attempted for the determination of the ideal stability limit, while also linking the features of this model with respect to their importance. We are proposing such an approach, showing that the original normalized beta, $\beta_{N}$, aspect ratio , $A$, internal inductance, $l_{i}$, and pressure peaking, $p_{0} /\langle p\rangle$, are among the parameters that most affect the estimated values of $-\delta W_{n o-\text { wall }}^{n=1}$, as expected by the underlying physics. Therefore, strengthened by this analysis, we have subsequently used a neural network to obtain an improved equation for the no-wall $\beta$ limit. Stability regions can be evaluated by examining plots of $-\delta W_{n o-\text { wall }}^{n=1}$ in the $2 \mathrm{D}$ parameter spaces of $\beta_{N}$ vs. $l_{i}, \beta_{N}$ vs. $p_{0} /\langle p\rangle$ and $\beta_{N}$ vs. $A$. Then neural network defined decision boundaries determine the marginal stability boundaries, increasing the accuracy compared to the previously defined no-wall limit modeling [7]. The overall no-wall beta limit input into the kinetic model can be determined by combining the defined boundaries with the dependencies of $\beta_{N, n o-\text { wall }}^{n=1}$ on the parameters above used in the neural network based model.

The remainder of the paper is organized as follows. In Section 2 the DECAF code is described, in Section 3 we review the physics of a specific module in it which models the ideal global plasma stability. In Section 4 the first machine learning technique, the RFR, is utilized to get estimated values of $-\delta W_{n o-\text { wall }}^{n=1}$. Section 5 describes the results of the neural network approach to defining decision boundaries between the stable and unstable regions in plasma parameter space. These are consequently integrated into the DECAF code in Section 6, and then applied for cross-machine testing to another spherical tokamak, MAST, in section 7. Finally, Section 8 concludes the paper. 


\section{Physics-guided ML approaches for ideal stability forecasting}

\section{The Disruption Event Characterization and Forecasting (DECAF) Code}

Tokamak plasma confinement devices utilize magnetic fields to contain high pressure plasmas for fusion energy. The plasma creates a component of its own confining magnetic field by carrying a large toroidal current. If the current is disrupted, a loss of plasma confinement results, which can lead to large heat deposition and electromagnetic forces on the surrounding structures. These so-called disruptions [30, 31, 32] have varying causes and must be avoided for the safe operation of future fusion devices including ITER. Some of the major causes of disruptions in tokamaks are: when the plasma density, plasma current, or ratio of plasma pressure to magnetic pressure exceed empirical or theoretically defined limits, or when the plasma loses vertical position stability and drifts into contact with surrounding surfaces [33]. A framework has emerged which provides a comprehensive approach to disruption prevention through forecasting and avoidance, or prediction and mitigation of the detrimental consequences. First, it is important to identify disruption event chains and the specific physics elements that comprise those chains. Second, if the events in the disruption chains can be forecast, cues can be provided to an avoidance system to attempt to break the chain. Multiple avoidance actions can be taken through available actuators, giving priority to events that tend to happen earlier. Finally, if avoidance is deemed untenable, a prediction of the impending disruption can be provided to a mitigation system to significantly reduce disruption ramifications. Designs for disruption mitigation systems for ITER are already under way $[34,35,36]$. Alternative disruption avoidance approaches also exist, including for example real-time plasma state estimation $[37,38]$ or by compiling large databases of previous disruptive discharge data [39, 40] and training various machine learning techniques $[41,42,43,44,45]$ on them, including the previously mentioned neural networks or random forests.

In the present work, we use the Disruption Event Characterization and Forecasting (DECAF) code, which utilizes the comprehensive structure described above. The ultimate goal of such an approach is to provide forecasts which integrate with a disruption avoidance system and are used in real-time during a device's operation. As shown in Figure 1, this approach provides a flexible framework to evaluate the proximity of plasma states to detected disruption events by coupled physics analyses, model criteria, and machine learning techniques.

Although machine learning approaches are often referred to as black box techniques, this definition is actually quite misleading in general. Neural networks have substantial deficiencies when they treat the system to be modeled as a black box, rather than making any assumptions about its behaviour, especially when attempting to use resulting neural network matrices for forecasting and extrapolation outside the training domain. In other words, the development of a shallow/deep learning system is complex, but does not need to be a black box. Machine learning in DECAF, by contrast, follows a philosophy that is more amenable to produce human understanding of the results and allows greater flexibility for use in control systems. Specifically, we have presently adopted three 
1

2

3

4

5

6

7

8
Physics-guided ML approaches for ideal stability forecasting

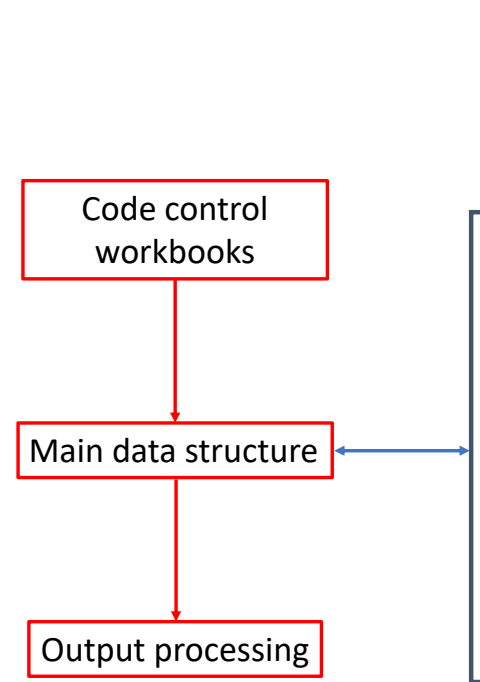

Output processing

Figure 1: The Disruption Event Characterization and Forecasting framework illustrating physical event modules.

general approaches:

(i) Reduction of results from certain complex physical models by shallow ML algorithms to allow rapid (including real time) determination of quantities used in DECAF models.

(ii) Hybrid model approach that combines the use of a physical model and ML techniques. The benefits are twofold: on the one hand, ML can be applied to produce the part of the problem that does not have a clear physical model (akin to an observer calculation in control theory); on the other, physics insight can be used to regularize a ML algorithm.

(iii) Analysis of DECAF event chain linkages pertaining to disruption prediction and avoidance understanding, utilizing graph theory [46]. This approach can give a mathematical representation of the event chains and can be used to process and interpret data structures in large datasets of DECAF events, in order to gain knowledge on how they group and what are their key relationships.

The analysis presented later in this paper falls mainly into the first category above, while also touching upon the second, which is an important new frontier in machine learning application to physics problems.

\section{Ideal MHD stability in the NSTX Spherical Tokamak}

Global magnetohydrodynamic (MHD) stability of high performance tokamak plasmas has long been recognized as a requirement for fusion-grade applications. Tokamak 


\section{Physics-guided ML approaches for ideal stability forecasting}

fusion plasmas are theoretically stable up to a value of the ratio of plasma stored energy to magnetic confining field energy of $\beta_{N, n o-w a l l}^{n=1}$. With ideal theory, the plasma is unstable above this "no-wall" limit when no electrically conducting wall is present to external kink-ballooning modes driven by the free energy of current of pressure gradients. Successful wall stabilization of kink/ballooning instabilities uncovered the reduced growth rate, yet still disruptive, resistive wall mode (RWM) [47, 48, 49, 50]. The RWM grows on a much slower time scale, the wall-time $\tau_{w}$, but it is still fast compared to the duration of the plasma shot, and is still theoretically unstable above the no-wall limit with ideal theory. Because the fusion power and the self-generated current in a tokamak rise with $\beta$, it is strongly desirable to operate at high $\beta$ above the no-wall limit. Therefore it is necessary to stabilize the RWM as well. Tokamak experiments found the fortuitous result that plasmas could be stably operated above $\beta_{N, \text { no-wall }}^{n=1}[51,52]$ with passive stability, not active control. Understanding the physics of this stability is key to relying on it and projecting it to the operation of future devices.

One of the functions of the DECAF code is to monitor fusion plasma's stability and predict when those plasmas might be approaching instability so that something can be done to avoid a catastrophic loss of plasma confinement. In the present paper we will examine data from the National Spherical Torus Experiment (NSTX), which was a spherical tokamak that operated at low aspect ratio and high beta. Disruption of the plasma current leading to thermal and current quenches, halo currents $[53,54]$, and heat and electromagnetic forces on the device structure were tolerated in NSTX experiments as the energy in the disruptions was not high enough to cause damage to the device. A reduced kinetic model for resistive wall mode stability based upon theoretical understanding has already been included in DECAF [2]. Here we will briefly describe the kinetic model and its underlying ideal stability components.

Calculation of the complex global mode frequency in a plasma, $\omega$, can be performed through the energy principle approach of calculating changes in potential energy $(\delta W)$ due to various effects and inputting these into a dispersion relation for $\omega$ (or stability criteria for the growth rate $\gamma$ ). $\mathrm{Hu}$ and Betti derived a modified energy principle for RWMs that includes the kinetic contributions of particle motions [4, 55]. The resulting dispersion relation is:

$$
\left(\gamma-i \omega_{r}\right) \tau_{w}=-\frac{\delta W_{n o-w a l l}^{n=1}+\delta W_{K}}{\delta W_{\text {with-wall }}^{n=1}+\delta W_{K}}
$$

where $\omega_{r}$ is the real frequency of the mode, $\delta W_{n o-w a l l}^{n=1}$ and $\delta W_{\text {with-wall }}^{n=1}$ are fluid, or ideal, changes in potential energy terms where the stabilizing device conducting structure is omitted or considered, respectively, and $\delta W_{K}$ is the kinetic term. Therefore, inclusion of kinetic effects represents a modification to ideal stability [7]. The fluid terms can be broken out into terms depending on the magnetic field line shape, and currentdriven and pressure-driven instability terms [56]. This is why measurable quantities such as $A, l_{i}$ (which is related to current profile peaking), $\beta_{N}$, and $p_{0} /\langle p\rangle$ are known to be influential on ideal MHD stability. The DCON stability code was developed to calculate 


\section{Physics-guided ML approaches for ideal stability forecasting}

the fluid $\delta W$ terms and was previously used for thousands of calculations spanning the NSTX operating space [7]. Analysis of these thousands of calculations formed the basis of an analytical model for the fluid $\delta W$ terms that was incorporated into the DECAF code [2]. Improvements to that model now by means of machine learning is examined in this paper.

Machine learning techniques, guided by physics, will be used as a tool in the present work to determine the best fit to ideal MHD stability limits, as calculated by DCON, within the range of parameters for which experimental data is available. In other words, the DCON calculations are the physics guidance within the range of applicability. However, as we will see, ML techniques can also extrapolate outside of the training region, and one must be cautious about accepting those projections at face value. In that case, plasma physics theory of ideal MHD will be used to guide the predictions. For example, it is well known that high pressure peaking is destabilizing to pressure-driven kink modes [57, 58, 59], and that Troyon criterion implies that the stability should decrease with aspect ratio $[57,60,61]$.

\section{Random Forest Regression for the value of $-\delta W_{n o-\text { wall }}^{n=1}$}

\subsection{Data pre-processing}

Major efforts have been devoted to modeling $-\delta W_{n o-\text { wall }}^{n=1}$ and $\beta_{N, n o-\text { wall }}^{n=1}$ by defining analytic relationships between plasma parameters [7]. This approach maintains simplicity and causal inference of the dependencies. However, the prediction of the value of $-\delta W_{n o-\text { wall }}^{n=1}$ can be improved further by including more plasma parameters and utilizing different approaches. Listed in Table 1, are 19 plasma parameters from the equilibrium reconstruction and various diagnostics that can be used as features to predict aspects of MHD stability.

Recent studies have focused on the possibility of combining machine learning algorithms with prior physics intuition [62, 63, 64]. For example, the process of preselecting the input parameters reduces the risk of redundancy while keeping physical significance. In this specific application, one can discern from the physics that the first 10 parameters listed above are the ones that are mostly correlated to $\delta W_{n o-w a l l}^{n=1}$. However, we must first make two important considerations. First of all, the toroidal rotation terms were missing for around 2000 data points and undermined the model performance, as shown later in Table 3. Second, when dealing with multivariate regression problems, it is also essential to check that the absolute value of the pairwise correlation between independent variables (predictors) is below a certain threshold, typically 0.7 [65]. Whenever two predictors are highly correlated with one another, both of them will have unstable partial regression coefficients with relatively large standard errors. This issue is usually referred to as multicollinearity and can compromise the statistical significance of a predictor, resulting in a higher potential for overfitting.

With regards to this, we have computed the pairwise Pearson correlation between 
Physics-guided ML approaches for ideal stability forecasting

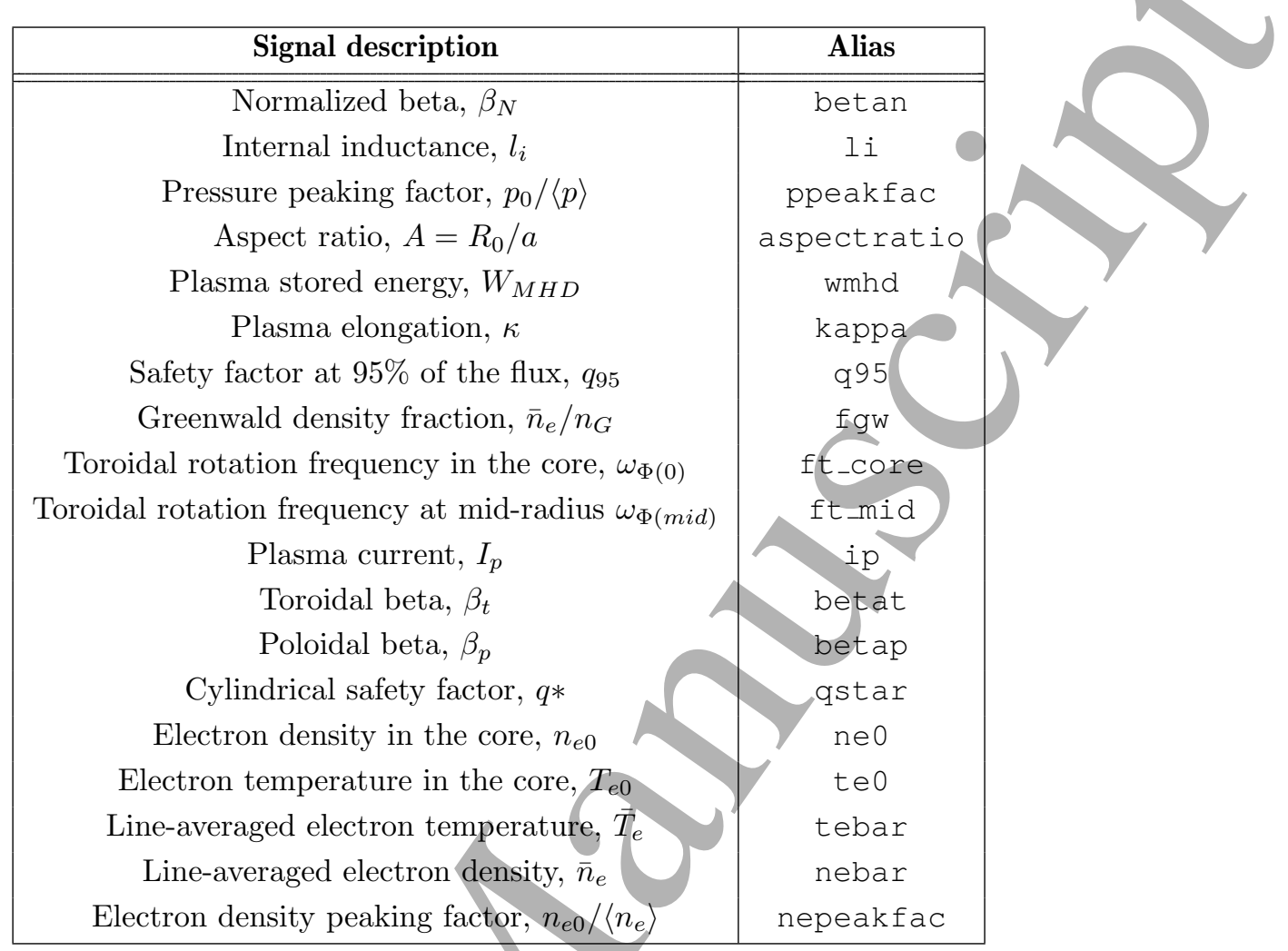

Table 1: List of signals used from NSTX equilibrium reconstructions and diagnostics. Left column shortly describes each signal, while the right column provides the alias name as it appears in the database.

the 8 most relevant predictors (excluding rotation terms) as shown in the colormap in Figure 2. This analysis shows evidence of multicollinearity between plasma parameters linked to the same underlying phenomena, such as $W_{M H D}$ with $\bar{n}_{e} / n_{G}$, and $A$ with $\kappa$. Such correlations are expected from constraints related to physical operation of the NSTX device. It is also worth noting that the correlation between pressure peaking factor and internal inductance is quite close to the 0.7 "limit". This experimental relationship was already known, since pressure broadens with broadened current profiles (see Figure 4 in J.W. Berkery et al. [7]). We have found that the two strong correlations introduced a minor overfitting issue, whereas leaving out either $p_{0} /\langle p\rangle$ or $l_{i}$ from the feature space led to worse performance, because of the high impact these plasma parameters have on the determination of $-\delta W_{n o-w a l l}^{n=1}$. The effect of correlated input variables will be analyzed in depth later.

\subsection{Hyperparameter tuning}

The RFR algorithm belongs to the ensemble methods class, building multiple decision trees and merging them together to get a more accurate and stable prediction. Each tree selects a random number of features in order to better differentiate the influence of each input parameter on the overall predictions. The final output is affected by several 


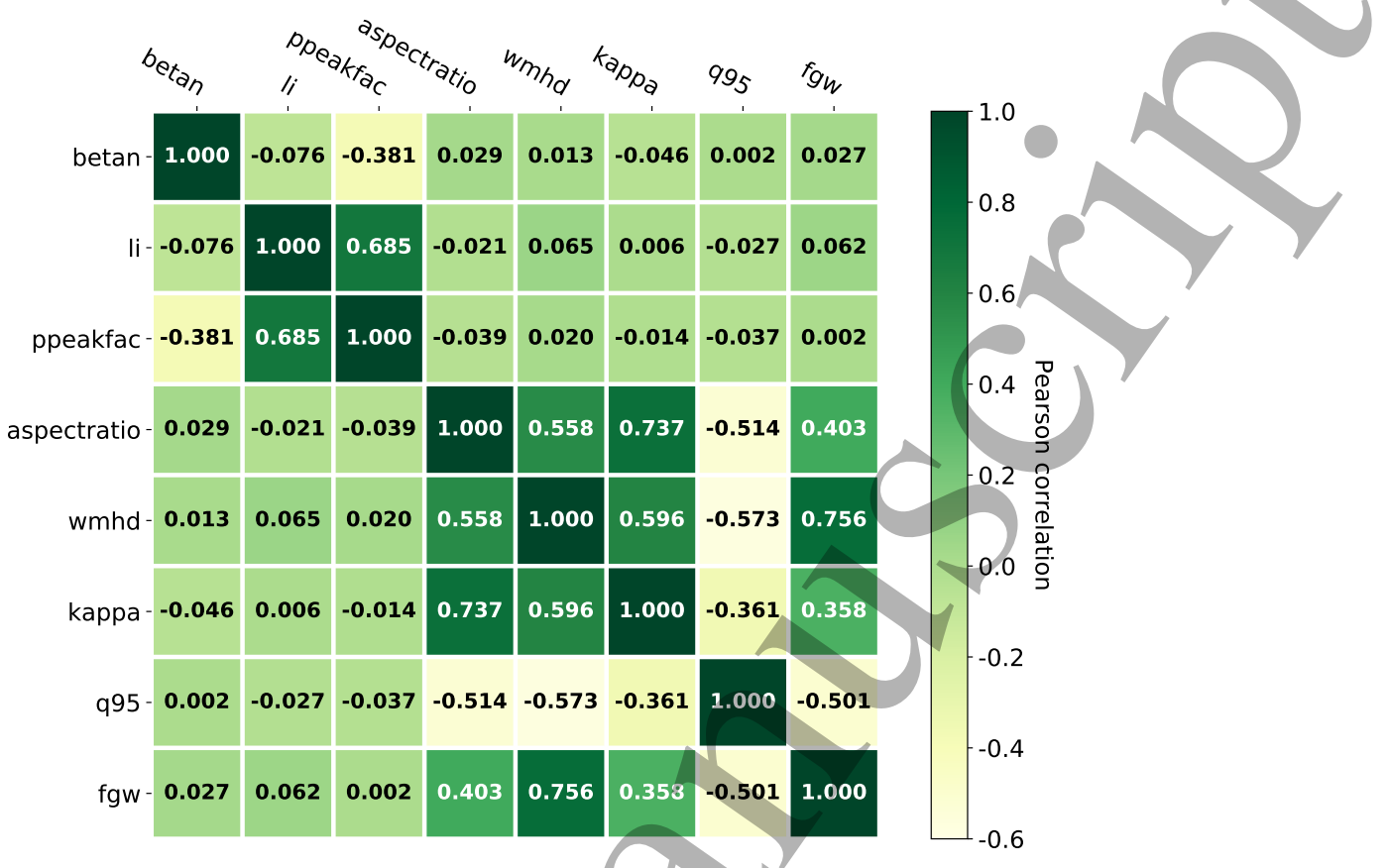

Figure 2: Pearson correlation matrix for the most relevant plasma parameters. A pairwise coefficient above 0.7 generally means that the two predictors are strongly correlated and may affect the model performance.

hyperparameters; among them the most important are the number of trees in the forest, the maximum number of features considered by each tree, the maximum number of levels in each tree, the minimum number of observations to split an internal node and the minimum number of observations to be at a leaf node (deepest level).

The random forest analysis has the advantage of high predictive power for non-linear regression problems and it is regarded as an interpretable technique, since it provides the importance of each input feature and allows to have an insight into decision paths via the export_graphviz function in the scikit-learn Python library (see figure 4 in C. Rea et al. [27]).

We have trained a RFR on a large dataset, comprising of 1385 shots for training and 293 for testing. Each shot contained around 5 measurements of $\delta W_{n o-w a l l}^{n=1}$, totalling more than 10000 data points for the entire set of available equilibria. The RFR has been tuned by running 5-Fold Cross Validation for 600 random combinations of the hyperparameters and for each of the tested models. As widely explained before, the performance of any machine learning algorithm is strongly affected by the choice of the input variables. The first model utilizing 8 plasma quantities to test the RFR resulted in a minor overfitting problem. Luckily, the RFR algorithm allowed us to analyze the relative importance of each feature (Table 2) and to discern which parameters we could sacrifice to reduce the chances of overfitting.

Therefore, based on relative importance, we have decided to drop out $\kappa$ and $\bar{n}_{e} / n_{G}$, solving the overfitting issue without compromising the generalization performance. As 


\section{Physics-guided ML approaches for ideal stability forecasting}

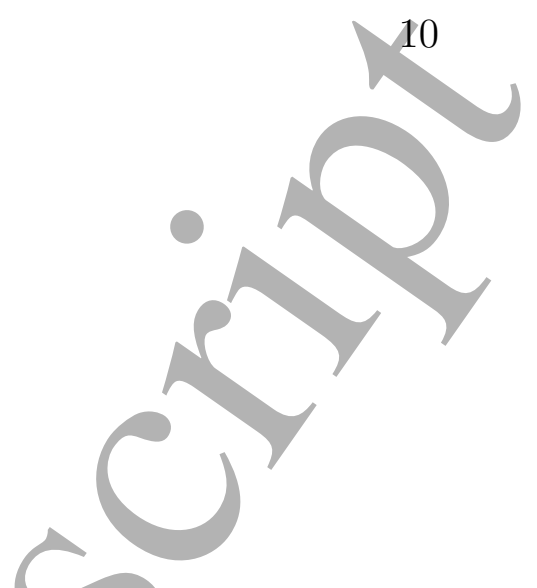

\begin{tabular}{|c|c|}
\hline Signal & Relative importance \\
\hline \hline betan & 0.633184 \\
li & 0.200685 \\
ppeakfac & 0.087245 \\
wmhd & 0.016737 \\
fgw & 0.015968 \\
aspectratio & 0.015762 \\
kappa & 0.015602 \\
q95 & 0.014817 \\
\hline
\end{tabular}

Table 2: Relative signal importance for the 8-feature RFR. The measure based on which the optimal conditions are chosen is called impurity. For regression trees, this is typically the variance. When training an entire forest, the decrease in impurity due to each feature can be averaged and the features are ranked according to this metric.

an additional test, we have also tried to train the random forest excluding either the pressure peaking factor or the internal inductance. Table 3 shows the results obtained with each of the mentioned combinations in terms of coefficient of determination, $R^{2}$, as well as the performance of the original reduced model [7]. Here one can get an idea of the importance that $p_{0} /\langle p\rangle$ and $l_{i}$ have in the determination of $\delta W_{n o-\text { wall }}^{n=1}$, despite the correlation between the two plasma quantities.

\begin{tabular}{|c|c|c|}
\hline & \multicolumn{2}{|c|}{ Coeff. of determination $\left(\mathbf{R}^{2}\right)$} \\
\cline { 2 - 3 } & Training set & Testing set \\
\hline \hline Original reduced model & - & 0.344 \\
RFR - 10 features & 0.789 & 0.735 \\
RFR - 8 features & 0.822 & 0.776 \\
RFR dropping $\kappa$ and $\bar{n}_{e} / n_{G}$ & $\mathbf{0 . 7 8 6}$ & $\mathbf{0 . 7 7 5}$ \\
RFR dropping $l_{i}$ only & 0.621 & 0.602 \\
RFR dropping $p_{0} /\langle p\rangle$ only & 0.711 & 0.682 \\
\hline
\end{tabular}

Table 3: Performance of the previous model and the four combinations of plasma parameters input to the RFR, with the best chosen regressor highlighted in bold. The original reduced model was directly validated on the entire dataset, therefore there is no distinction between training and testing set.

The best selected model had 600 estimators (trees), each one having a maximum of 15 levels. These (and other hyperparameters) were chosen on the basis of the lowest cross-validation mean squared error. The relative importance of the input features is displayed in Table 4 and reflects, similarly to the 8-feature analysis, what we expected from the underlying physics.

The DCON computed $-\delta W_{n o-\text { wall }}^{n=1}$ vs. the RFR predicted value as a function of equilibrium quantities is plotted in Figure 3. The left hand plot (a) displays the results 
1

2

3

4

5

6

7

8
Physics-guided ML approaches for ideal stability forecasting

\begin{tabular}{|c|c|}
\hline Signal & Relative importance \\
\hline \hline betan & 0.688461 \\
li & 0.198174 \\
ppeakfac & 0.074881 \\
wmhd & 0.014078 \\
aspectratio & 0.013183 \\
q95 & 0.011223 \\
\hline
\end{tabular}

Table 4: Relative signal importance for the 6 -feature regression.

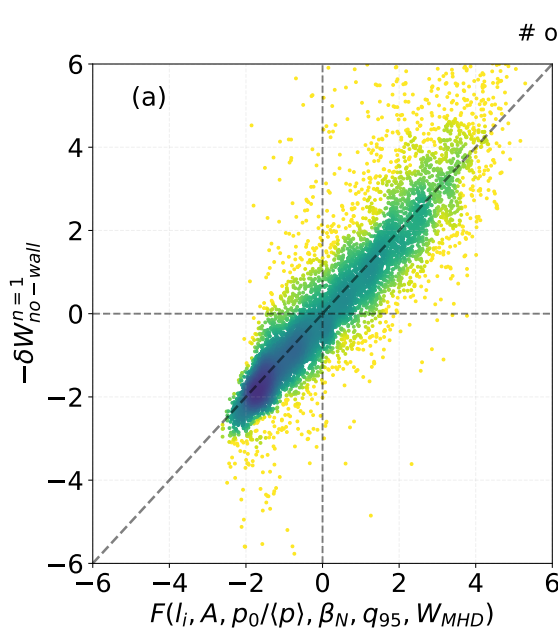

\# of samples

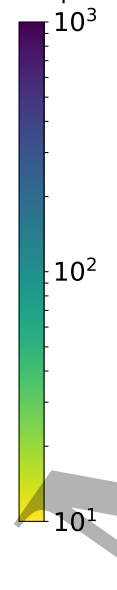

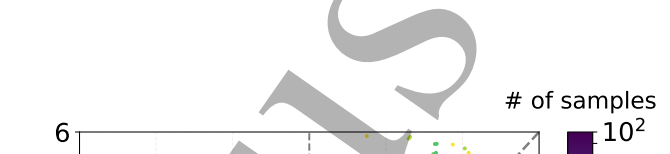

(b)

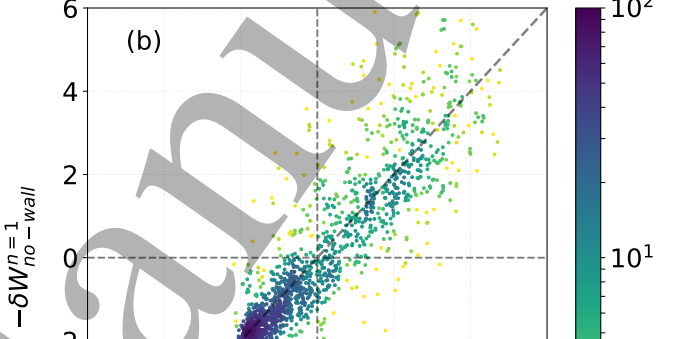

$1-2$

Figure 3: $-\delta W_{n o-w a l l}^{n=1}$ computed by DCON vs. $F\left(l_{i}, A, p_{0} /\langle p\rangle, \beta_{N}, q_{95}, W_{M H D}\right)$ predicted by the 6 -feature RFR for the (a) training and (b) testing sets. Each data point is colorcoded based on the spatial density of nearby points. Therefore, darker regions indicate higher density, whereas lighter colors indicate higher sparsity of the points.

obtained on the training set, whereas the plot on the right (b) shows the predictions during the test phase. Each point is colored by the spatial density of nearby points, spanning from dark blue (high density) to yellow (low density). In both cases there is generally a linear correspondence with some spread.

Furthermore, as long ás $-\delta W_{n o-w a l l}^{n=1}$ can take both negative and positive values, we noticed that the stable/unstable classification improves with increasing regression performance. Therefore, we can compute the percentage of misclassified points (e.g. the ones in the upper left and bottom right quadrants of Figure 3(a),(b)), even though the RFR is performing a regression task. In this case, the RFR classifies points better than the previous model, reaching an overall accuracy of $91.1 \%$ in test phase and producing only $2.8 \%$ of false negatives and $6.1 \%$ of false positives, compared to the original reduced models $11.2 \%$ and $5.6 \%$, respectively.

Ultimately, we will now proceed to sacrifice performance somewhat in favor of an improved closed form equation for the no-wall limit to be used in the kinetic model. 


\section{Physics-guided ML approaches for ideal stability forecasting}

\section{Neural network defined decision boundaries for the no-wall limit}

\subsection{Base model configuration}

The random forest approach can provide the value of $-\delta W_{n o-\text { wall }}^{n=1}$ with a relatively high level of accuracy, but complications occurred when trying to use the RFR predictions to get an improved expression of the analytic equation for $\beta_{N, n o-w a l l}^{n=1}$ already inchuded in DECAF. We attempted to train a linear regressor on the input data resulting in RFR predictions of $\left|\delta W_{n o-\text { wall }}^{n=1}\right|<0.1$ to find the hyperplane that divides stable and unstable regions, but the two stacked regressors did not provide any improvement when compared to the original reduced model. Another possibility could be to choose a cross section of the 6-dimensional grid and project it down to 2D. However, the decision boundary of a multi-dimensional model is complex and any $2 \mathrm{D}$ projection will not represent the regressor/classifier in its entirety. More accurate Voronoi-based representation methods exist [66], allowing the visualization of multi-dimensional decision regions in 2D. Furthermore, high-dimensional decision boundaries can be characterized by combining adversarial example generation and PCA [67]. However, as a first step we have decided to exploit the knowledge given by the RFR in a different way to re-define an analytic expression for $\beta_{N, n o-\text { wall }}^{n=1}$.

A 3-layer fully connected neural network (Figure 4) is proposed to classify whether an equilibrium data point from NSTX is below or above the stability limit. The $j^{\text {th }}$ layer implements a linear operation that is activated through a logistic function, mapping the input to the subsequent layer $\left(X_{j+1}\right)$ into a range between 0 and 1 as follows:

$$
X_{j+1}=g\left(\Theta_{j} X_{j}+b_{j}\right)
$$

where $g$ is the logistic (or sigmoid) activation, $\Theta_{j}$ is the matrix of weights mapping from layer $j$ to $j+1, b_{j}$ is the bias term and $X_{j}$ is the feature vector input to the $j_{t h}$ layer. The dataset has been split in the same way as it was done for the random forest for direct comparison. The entire training set is iteratively scanned for 1000 times (epochs) and both the weights matrices and biases are updated at each pass.

We have trained different neural network architectures, varying the number of layers between 2 and 5 and the number of neurons between 8 and 128. The main goal was to find the best compromise between validation accuracy and physical explainability, reducing complexity in order to avoid the risk of overfitting. The learning phase was performed by separately training three identical neural networks with inputs $\left(\beta_{N}, l_{i}\right)$, $\left(\beta_{N}, p_{0} /\langle p\rangle\right)$ and $\left(\beta_{N}, A\right)$, respectively.

By preselecting these particular plasma quantities we are engaging in the practice of feature engineering, which is to try to feed the predictive model the data most representative of the problem, rather than the most raw data. The features are not only selected to make the most straightforward comparison to previous models, but are also the ones contributing with around $97 \%$ of the information according to the random forest. Each feature is Min-Max normalized since neural networks can better process values between 0 and 1 . The network was trained using a stochastic gradient 


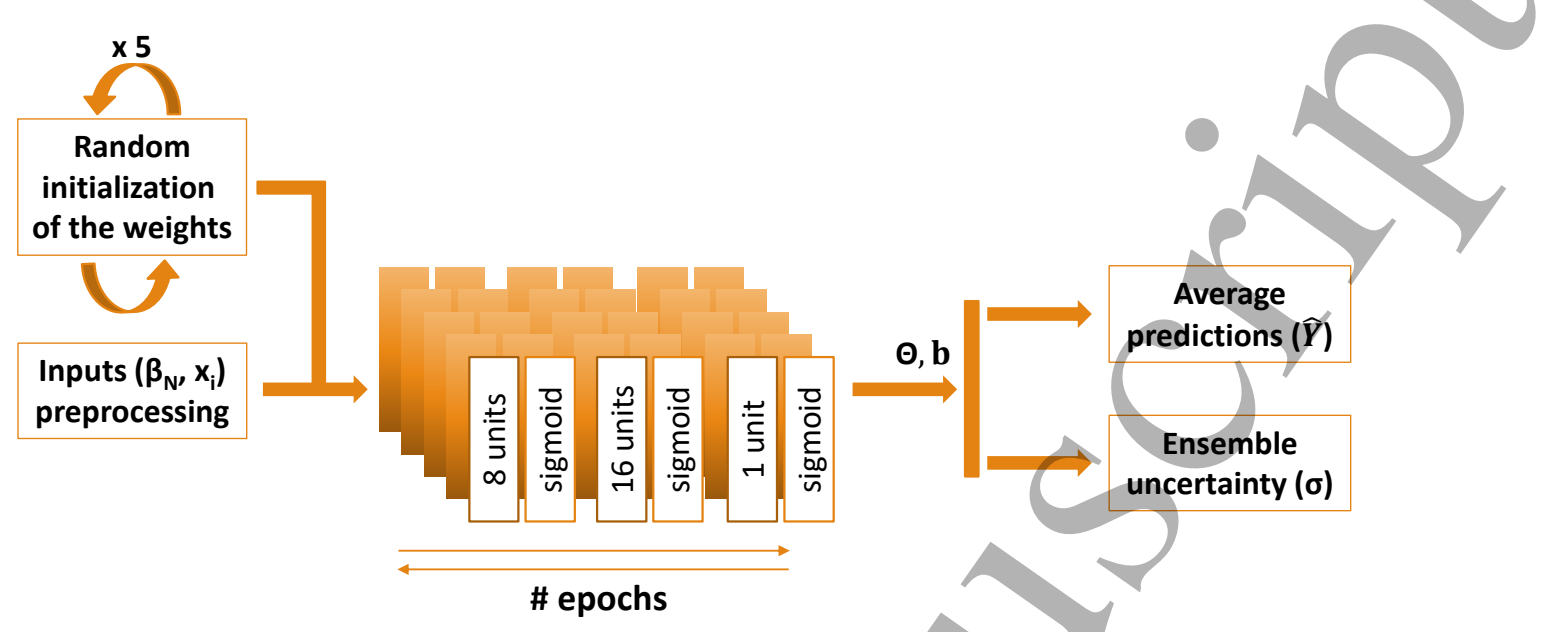

Figure 4: Proposed architecture of the ensemble neural network to classify whether a data point is below or above the no-wall stability limit in the three separate $\left(\beta_{N}, x_{i}\right)$ spaces, where $x_{i}$ is either $l_{i}, p_{0} /\langle p\rangle$ or $A$. The 5 sub-models outputs are amalgamated into an ensemble in order to evaluate the uncertainty on the predictions.

descent optimizer with decaying learning rate in the KERAS library [68] in order to find the optimum combination of parameters that minimizes the binary cross-entropy loss function, $\mathcal{L}[69]$.

Since neural networks can make different decisions depending on how the starting kernels are chosen, we have decided to build an ensemble of 5 models for each input, each taking a random initialization of the network weights. Similarly to what was done by M.D. Boyer et al. [23], the output is chosen to be the mean of the sub-models predictions and the standard deviation from the mean is utilized to provide a sense of the uncertainty on the predictions. The neural network returns the probability of $\delta W_{n o-w a l l}^{n=1}>0$ (i.e. the probability of belonging to the unstable class). Therefore, points having an output probability $<0.5$ are considered below the stability limit, while the ones above are classified as likely unstable points. The no-wall limit is defined as the locus of points (here decision boundary) where the neural network's outputs are equal to 0.5 .

\subsection{Physics-guided objective function}

We found that if the training phase was performed on a data-driven-only basis, a somewhat surprising result was that the no-wall $\beta$ limit would monotonically increase with pressure peaking, internal inductance and aspect ratio across the experimental domain. As was noted in Section 3, the theoretical expectation is that at higher pressure peaking (and $l_{i}$, the two parameters are correlated in NSTX [7]) the no-wall limit should decrease. To a lesser extent, the same is true for the aspect ratio projection, which should also be slightly decreasing rather than the implied slight increase, at higher A. Therefore, the theoretical physics guidance is imposed by modifying the learning 


\section{Physics-guided ML approaches for ideal stability forecasting}

objective function $[62,63]$ for the decision boundary projection at higher $p_{0} /\langle p\rangle, l_{i}$ and $A$. Normally, neural networks aim to minimize an empirical loss while keeping a low model complexity for better generalization. However, this approach doesn't guarantee that the predictions will be in line with the expected underlying physics, especially outside the domain of applicability. Therefore, we introduce an additional term to the loss function which penalizes the predictions with increasing $p_{0} /\langle p\rangle, l_{i}$ and $A$. Let us denote with $\hat{Y}$ the model predictions and with $Y$ the actual observations, and evaluate the new learning objective as follows:

$$
\underset{\Theta, b}{\arg \min } \underbrace{\mathcal{L}(\hat{Y}, Y)}_{\begin{array}{c}
\text { Empirical } \\
\text { loss } \\
\text { function }
\end{array}}+\underbrace{\frac{1}{N} \sum_{n=1}^{N} \frac{H\left(x_{i}\right)_{n}}{\hat{y}_{n}}}_{\begin{array}{c}
\text { penalization } \\
\text { term }
\end{array}}
$$

where $\mathrm{N}$ is the number of training samples and $H\left(x_{i}\right)$ is a two-step Heaviside function that defines the relative importance of the penalization term and it is an additional hyperparameter that needs to be properly chosen for each plasma quantity. In fact, an overly large step function would excessively penalize the predictions, resulting in a too negative steepness of the boundary at high $p_{0} /\langle p\rangle, l_{i}$ and $A$, whereas a small $H$ would not provide any change in the predictions. Therefore, we have randomly initialized for 20 times the magnitude of the two steps: the first was chosen in the range $[0.05,2]$ and the second one was constrained to be larger than the first but less than 4 . We have selected the values at which the boundaries smoothly decreased outside of the training region without compromising the validation accuracy within the points. The magnitude of $H$ for each of the plasma parameters is displayed in Table 5.

Stability regions and the uncertainty can be evaluated by plotting $\beta_{N}$ versus the other plasma quantities. Figure 5 shows the $2 \mathrm{D}$ space defined by $\beta_{N}$ vs. $l_{i}$, with each point representing a DCON calculation for an individual NSTX equilibrium color coded 
either in purple $\left(-\delta W_{n o-w a l l}^{n=1}<0\right.$, below the "no-wall" stability limit) or in yellow ($\delta W_{n o-w a l l}^{n=1}>0$, above). The contour plot is what the neural network predicts in each point of the grid in terms of probability. The plots include the physics-guided projections outside of the range where data was available; this point will be discussed further in Section 7.

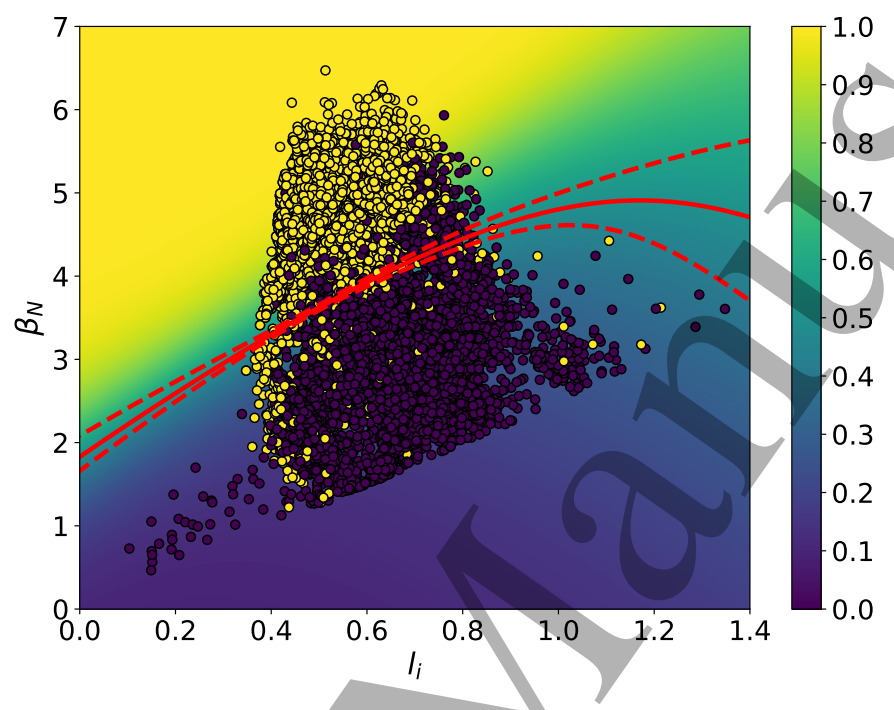

Figure 5: $\beta_{N}$ vs. $l_{i}$ space with the red solid line as the mean decision boundary and the dashed lines indicating the standard deviation from the mean. The contour plot shows the probability of being above the no-wall limit.

Highlighted as a red solid line is the decision boundary, which effectively defines the location of the no-wall beta limit. The red dashed lines indicate the standard deviation from the mean. As expected, the 5 sub-models make similar decisions where the density of the points is high, whereas they diverge outside the domain. An analytic expression approximating the no-wall limit can be obtained by fitting the best curve to the boundary points and in this case is given by:

$$
\beta_{N, b n d}\left(l_{i}\right)=4.91 e^{-\left(\frac{l_{i}-1.17}{1.14}\right)^{2}}+0.21 e^{-\left(\frac{l_{i}-0.27}{0.39}\right)^{2}}
$$

Similarly, Figures 6 and 7 show the decision boundaries for aspect ratio and pressure peaking, and the resulting equations are:

$$
\begin{gathered}
\beta_{N, \text { bnd }}(A)=-4.14 A^{3}+13.47 A^{2}-14.95 A+10 \\
\beta_{N, \text { bnd }}\left(\frac{p_{0}}{\langle p\rangle}\right)=2.56 e^{-\left(\frac{p_{0} /\langle p\rangle-4.06}{2.59}\right)^{2}} \\
+3.10 e^{-\left(\frac{p_{0} /\langle p\rangle-0.43}{4.29}\right)^{2}}
\end{gathered}
$$




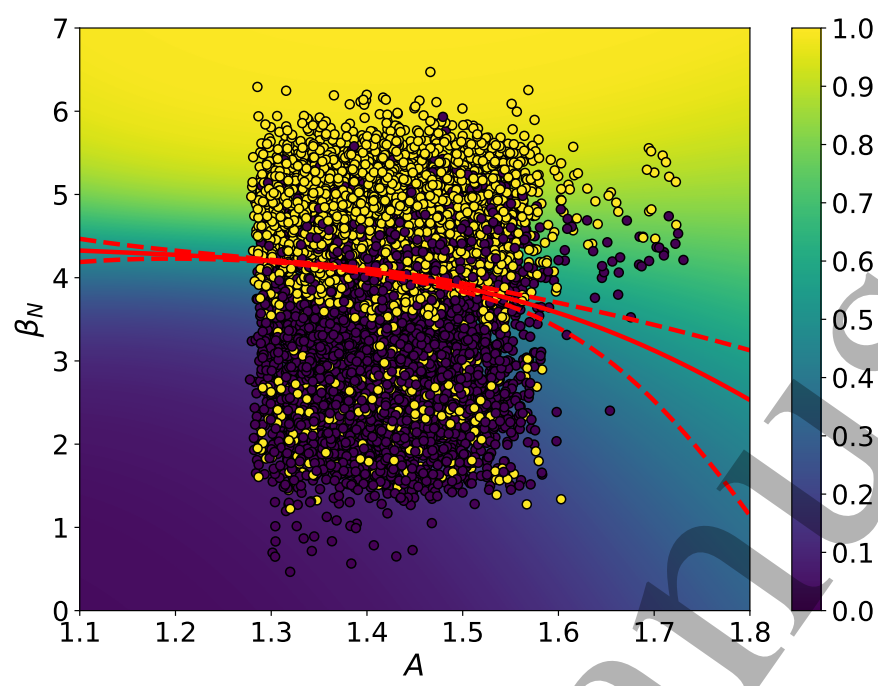

Figure 6: $\beta_{N}$ vs. A space for the NSTX database. Data points are color coded either in purple $\left(-\delta W_{n o-w a l l}^{n=1}<0\right)$ or yellow $\left(-\delta W_{n o-\text { wall }}^{n=1}>0\right.$.)

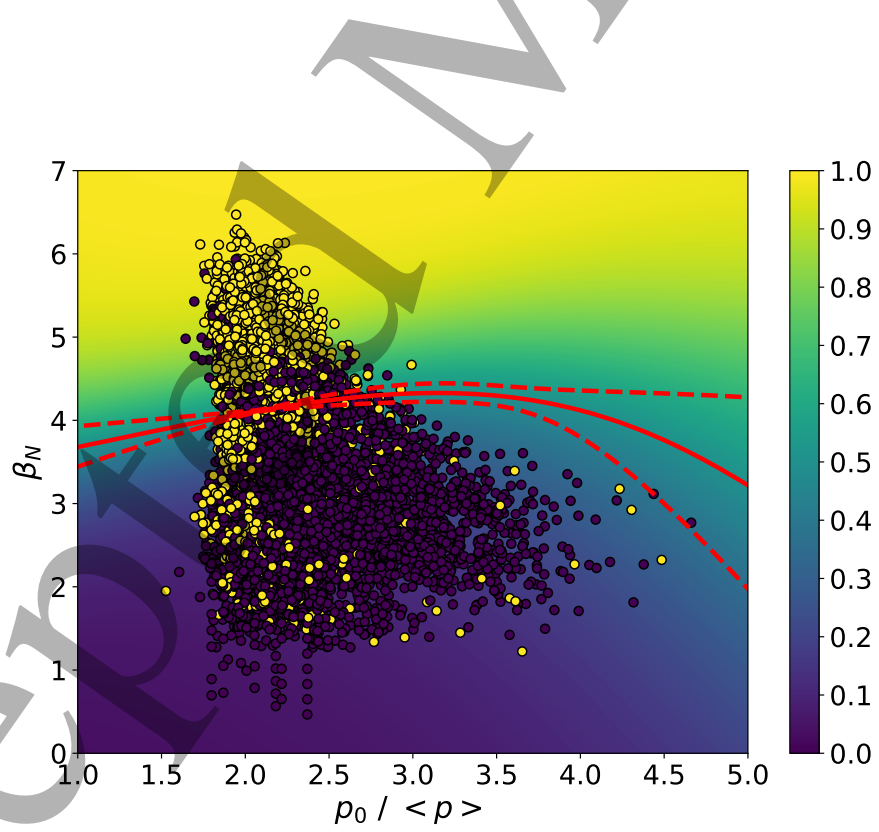

Figure 7: $\beta_{N}$ vs. $p_{0} /\langle p\rangle$ space for the NSTX database. Purple is below the no-wall limit (class 0) and yellow is above (class 1 ).

\subsection{Model performance and uncertainty quantification}

The overall no-wall beta limit can be determined by combining these defined boundaries in a way that we will briefly explain. First, by plotting $-\delta W_{n o-w a l l}^{n=1}$ vs. the ratio between 


\section{Physics-guided ML approaches for ideal stability forecasting}

$\beta_{N}$ and any of the other plasma parameters, it can be easily seen that the best fit is roughly given by a cubic expression (see Figure $2 \mathrm{~b}$ in J.W. Berkery et al. [7]). The same happens if we plot $-\delta W_{n o-\text { wall }}^{n=1}$ vs. $\beta_{N} / \beta_{N, b n d}\left(x_{i}\right)$. Secondly, as long as $\delta W_{n o-w a l l}^{n=1}$ must be equal to zero at the boundary, the best fit for each of the boundaries should be given by $-\delta W=a_{\beta_{N}, x_{i}}\left(\left(\beta_{N} / \beta_{N, b n d}\left(x_{i}\right)^{3}-1\right)\right.$; where the $a_{\beta_{N}, x_{i}}$ 's are coefficients that need to be optimized. Then, each $-\delta W$ term should be appropriately weighted via weighting factors (i.e. $w_{\beta_{N}, x_{i}}$ ) provided by the random forest. Therefore, the following fit for $F=$ $-\delta W_{n o-\text { wall }}^{n=1}$ will be used:

$$
F=a_{0}+\beta_{N}^{3} \sum_{x_{i}} \frac{a_{\beta_{N}, x_{i}} w_{\beta_{N}, x_{i}}}{\beta_{N, b n d}\left(x_{i}\right)^{3}}-\sum_{x_{i}} a_{\beta_{N}, x_{i}} w_{\beta_{N}, x_{i}}
$$

The chosen $a$ coefficients are the ones providing the highest $\mathrm{R}^{2}$ and accuracy. With regards to the weighting factors, one can see that in the above fit the importance of $\beta_{N}$ is implicit because it is included in each term. Moreover, since the $w_{\beta_{N}, x_{i}}$ 's should add up to 1 , they need to be rescaled based on the relative importance of $l_{i}, p_{0} /\langle p\rangle$ and $A$ from Table 4. Both the weights and the coefficients are listed in Table 6.

\begin{tabular}{|c|c|c|}
\hline & Weights & Coefficients \\
\hline \hline Intercept $\left(a_{0}\right)$ & - & -0.12 \\
$\beta_{N, l_{i}}$ & 0.3764 & 3 \\
$\beta_{N, p_{0} /\langle p\rangle}$ & 0.3246 & 1.2 \\
$\beta_{N, A}$ & 0.2990 & 1 \\
\hline
\end{tabular}

Table 6: Weighting factors and coefficients for the $\delta W_{n o-w a l l}^{n=1}$ fit.

Setting $F=0$ at the boundary, this results, finally, for an expression for the no-wall beta limit:

$$
\beta_{N, \text { no-wall }}^{n=1}=\sqrt[3]{\left[\left(\sum_{x_{i}} a_{\beta_{N}, x_{i}} w_{\beta_{N}, x_{i}}\right)-a_{0}\right]\left[\sum_{x_{i}} \frac{a_{\beta_{N}, x_{i}} w_{\beta_{N}, x_{i}}}{\beta_{N, b n d}\left(x_{i}\right)^{3}}\right]^{-1}}
$$

We can then propagate the error on the no-wall limit estimation by combining the uncertainty on the decision boundaries with the spread of $F$ around the zero. When the DCON calculated $\delta W_{n o-w a l l}^{n=1}$ is in the range $[-0.1,0.1]$, the neural network assisted predictions result in an average of $F_{0}=-0.06$ and a standard deviation $\sigma_{F}=1.05$. The resulting estimated uncertainty on $\beta_{N, n o-\text { wall }}^{n=1}, \sigma_{\beta_{N, n o-w a l l}}$, is obtained using the variance formula for the error propagation:

$$
\sigma_{\beta_{N, \text { no-wall }}}=\sqrt{\left(\frac{\partial \beta_{N}}{\partial F} \sigma_{F}\right)^{2}+\sum_{x_{i}}\left(\frac{\partial \beta_{N}}{\partial \beta_{N, b n d}\left(x_{i}\right)} \sigma_{\beta_{N, b n d}}\left(x_{i}\right)\right)^{2}}
$$

which translates into an error on the estimated no-wall limit of around $\pm 18 \%$. 

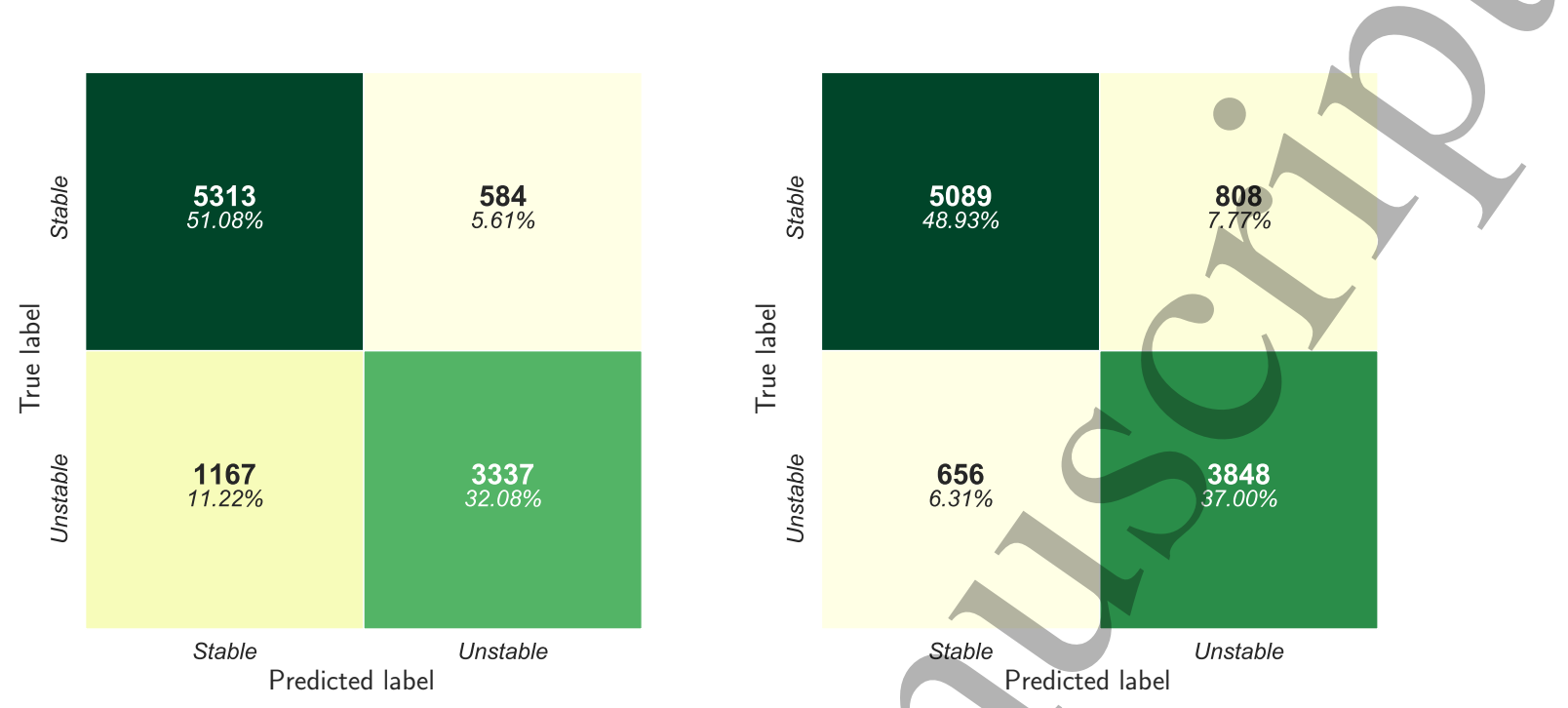

Figure 8: Confusion matrices for the (left) original and (right) neural network decision boundaries no-wall limit. The predictions are split into True Negatives (upperleft quadrant), False Positives (upper-right), True Positives (bottom-right) and False Negatives (bottom-left).

The analytic expression obtained has been tested at low $l_{i}<0.64$ and high $l_{i}>$ 0.64 , as well as at low $p_{0} /\langle p\rangle<2.25$ and high $p_{0} /\langle p\rangle>2.25$, for best comparison with the previously defined limit. The results are displayed in Table 7 , where accuracy indicates the percentage of the DCON calculations which fall on the correct side of the stable/unstable boundaries as defined by the original or neural network assisted boundaries. The results show that the neural network outperforms the previously defined equation in J.W. Berkery et al. [7], especially at low $l_{i}$, leading to an overall improvement in accuracy of around $2.8 \%$. The overall accuracy can also be displayed in a so-called "confusion matrix" where the classification is broken into quadrants based on the "actual" stable/unstable calculation from DCON, and the "predicted" stable/unstable model based classification.

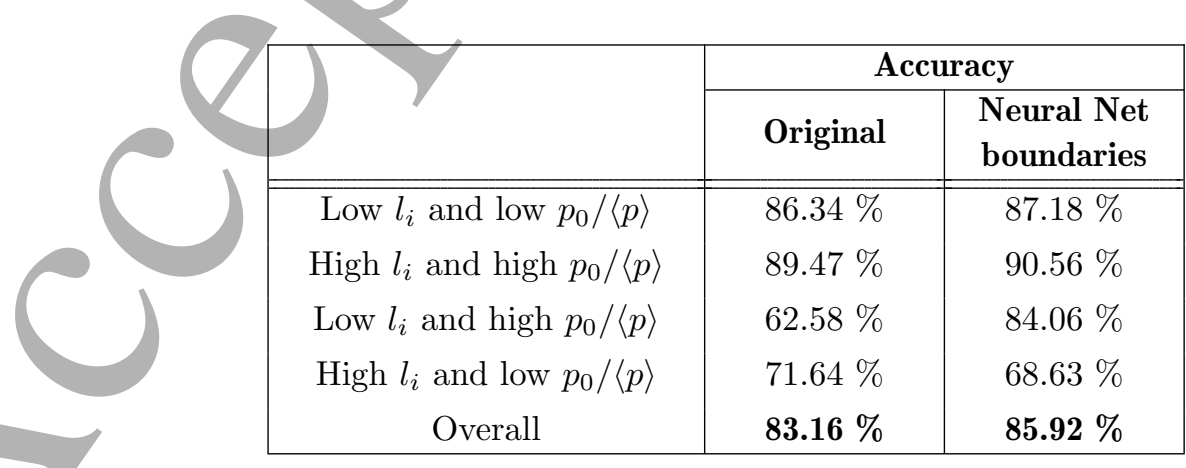

Table 7: Accuracy for the original splitting equations and for the neural network defined boundaries. 
These are shown in Figure 8 for the original and neural network analyses, respectively, where the overall accuracy numbers from the above table are the summation of the diagonal quadrants: calculated stable and predicted stable, and calculated unstable and predicted unstable. Here one can see that there is a slightly larger number of false positives, but with the benefit of an almost halved amount of missed instabilities (i.e. bottom-left quadrant in the confusion matrix). False positives, within certain limits, are acceptable, whilst an abundance of missed instabilities represents a risk for the safety of the reactor.

Overall, the neural network analysis is capable of finding decision boundaries that are still understandable in terms of a few expected plasma parameters and that perform better than the previous analytic technique in terms of classification accuracy . On the other hand, the 2D analysis intrinsically excludes possible correlations between features. The decisions the neural network makes could be stronger and more accurate by feeding it with all the parameters available, rather than just two at a time. However, this approach does not provide a closed form equation for the decision boundary to be included in the larger kinetic model. One possibility could be to use the 19-parameter space shown in Table 1 and apply a dimensionality reduction technique (i.e. Principal Component Analysis, t-SNE, etc.) to map the original features into a low dimensional space, find the decision boundary in this "high-level" space and then map it back to real plasma parameters.

\section{Integration of machine learning techniques into the DECAF global stability module}

The ultimate goal of the global stability monitoring algorithm is to include not only ideal, but also kinetic effects - which has been shown to be able to explain experimental stability [7]. A reduced model including kinetic effects is already implemented in DECAF, but there are a few possible ways to improve this approach. One would be to utilize machine learning techniques for the kinetic $\delta W_{K}$ terms in the way that we have done here for the ideal no-wall terms, by constructing a neural network or a random forest that can accurately approximate the results of a stability code calculation in a small fraction of time. Unfortunately, this technique works best when many thousands of code calculations are available, spanning the operational space of a device. This is not the case for kinetic calculations. For example, the MISK code has been used to determine the kinetic stability of NSTX for many equilibria, but due to the more demanding computational nature of the code, compared to DCON for example, the number of available MISK runs is certainly not thousands, let alone hundreds. This is why an analytic reduced kinetic model was originally developed [1].

Second, it is possible to run the present analytic reduced model on a very large set of discharges to obtain a database of calculated kinetic growth rates to be compared to experimental stability. Then, a machine learning technique could replicate that analysis producing stability maps and defining paths for improvement. One key question to 


\section{Physics-guided ML approaches for ideal stability forecasting}

be demonstrated by such an approach would be how smoothly the gradients and the partial derivatives flow in the stability map with various parameters. This is a necessary line of research for a machine learning technique that will ultimately be interfaced into a control system. Control algorithms will fail if machine learning techniques can not provide smooth and reliable stability maps, upon which to act. The research line just described will be the subject of future work.

\subsection{Application to post-discharge NSTX data}

For the present purposes, a less ambitious approach is to utilize the reduced kinetic model for $\gamma \tau_{w}$ (as in 1) currently included in DECAF, but with the neural network improved calculation of the $\beta_{N, n o-\text { wall }}^{n=1}$ term (i.e. using 8). In this case we anticipate small changes, but adding slightly more accuracy, to the computed growth rate.

Figure 9 shows a comparison of the two models for the normalized ideal fluid growth rate in black, and the full kinetic models as colored solid lines versus time leading to disruption, for NSTX shot 139514.

Figure 9: Normalized ideal fluid growth rate vs. time to disruption in black dashed line for the original DECAF model, and black solid line using the NN boundaries, for NSTX 139514. The colored lines indicate the original and modified kinetic growth rates in red and green, respectively.

One can see that the solid black line, indicating the neural network modeling 
of $\gamma \tau_{w, \text { fluid }}$, gives smaller values than the current DECAF model (dashed). This is mainly due to the reduced amount of missed instabilities (false negatives) in the

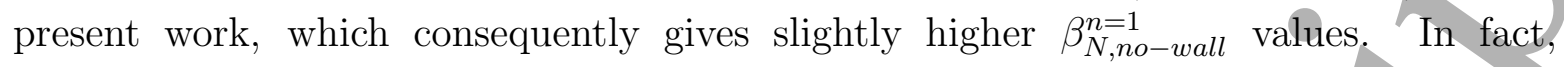
since the ideal growth rate is computed as a function of the familiar parameter $C_{\beta}=\left(\beta_{N}-\beta_{N, \text { no-wall }}^{n=1}\right) /\left(\beta_{N, \text { with-wall }}^{n=1}-\beta_{N, \text { no-wall }}^{n=1}\right)$, the previous observation reflects in lower $C_{\beta}$ values, with a smaller $\gamma \tau_{w}$,fluid for the entire shot as a direct consequence.

This decrease in $\gamma \tau_{w, \text { fluid }}$ propagates in the green line as well, where the kinetic growth rate is shown as a moving average for illustrative purposes. It is worth emphasizing that all the moving averages in DECAF use only previous time points, in order to be applicable in future real-time disruption warning systems. Again, no big changes have been obtained in the final DECAF output, although in this particular case, the improved $\gamma \tau_{w \text {,kinetic }}$ has avoided a possibly erroneous early zero-crossing (i.e. when the model estimates the RWM is going unstable).

When applied to a set of NSTX discharges previously analyzed by the DECAF kinetic model [1], the current physics-guided, machine learning assisted model failed to predict an unstable RWM in 3 out of 20 experimentally unstable discharges, whereas just one out of 9 stable discharges was predicted to be unstable. The fact that improving one component in the dispersion relation (Eq. 1) leads to results consistent with current DECAF calculations is quite promising and lays the basis for further machine learning assisted computations of the $\delta W_{K}$ term.

\subsection{Simulated real-time stability calculations}

It was previously stated that one benefit of training machine learning algorithms on databases of calculations is their ability to emulate those calculations much more quickly given the inputs. Tokamaks generally have some measurements and analyses, such as equilibrium reconstruction, available in real-time during the discharge. Real-time disruption avoidance algorithms can take advantage of that by using these inputs and trained machine learning algorithms to provide stability quantities faster than the realtime operation of the plasma device. In this case we can simulate what the realtime $\beta_{N, n o-w a l l}^{n=1}$ and $-\delta W_{n o-w a l l}^{n=1}$ from the neural network would have looked like for an NSTX discharge, using only the inputs that were available in real-time for that shot. Then, we can compare these quantities to the no-wall limit from the DECAF algorithm and $-\delta W_{n o-\text { wall }}^{n=1}$ from a DCON calculation, which use post-processed equilibrium reconstructions as input.

Figure 10(a) shows in black the real-time equilibrium reconstruction of $\beta_{N}$, with the simulated real-time no-wall limit in red and the $\pm 18 \%$ error bar as a grey shaded area, for NSTX discharge 138556. Frame (b) displays the emulated $-\delta W_{n o-w a l l}^{n=1}$ using Eq. 7 along with the error bars in grey. The DCON computed $-\delta W_{n o-w a l l}^{n=1}$ versus time from post-discharge analysis is plotted in Fig. 10(c).

The neural network simulation of real-time $\beta_{N, n o-\text { wall }}^{n=1}$ and $-\delta W_{n o-\text { wall }}^{n=1}$ indicates that the plasma is above the no-wall stability limit for $0.4-1.0 \mathrm{~s}$. The value of a real-time 
Physics-guided ML approaches for ideal stability forecasting

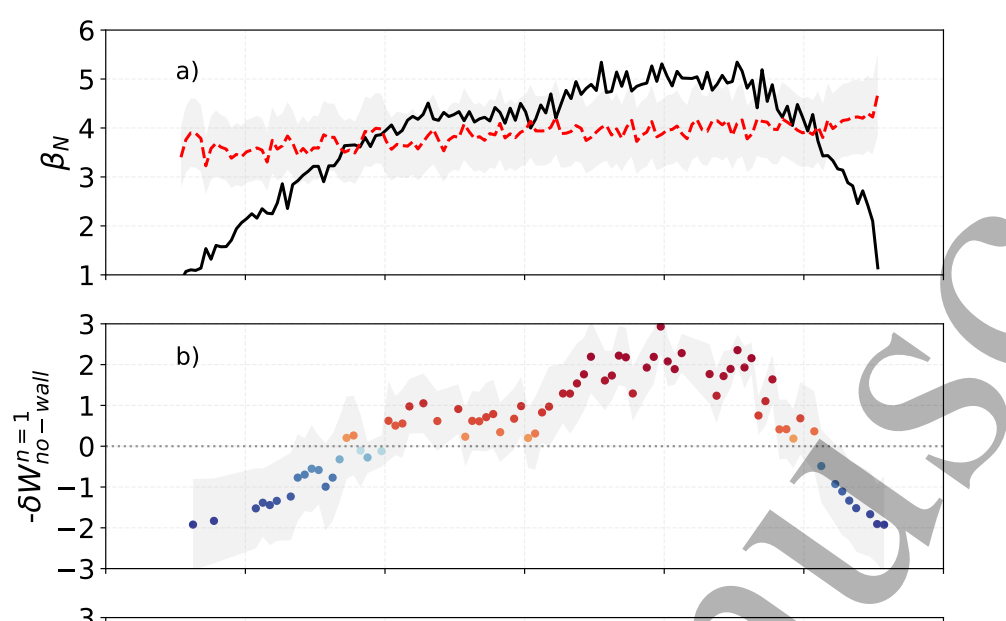

Figure 10: Simulated real-time calculations for NSTX discharge 138556. (a) $\beta_{N}$ vs. time with the estimated no-wall limit and the uncertainty area shown in red and grey, respectively. (b) Estimated $-\delta W_{n o-\text { wall }}^{n=1}$ and the $\sigma_{F}$ as a grey shaded area. (c) DCON computed $-\delta W_{n o-w a l l}^{n=1}$ (ground truth).

calculation of $\delta W_{n o-w a l l}^{n=1}$ has been recognized [10], and these alarms will be used in a future DECAF real-time disruption monitoring algorithm. In fact, even if the plasma remained experimentally stable in this particular case, other unintended consequences can occur when a plasma crosses the no-wall limit, such as error field amplification and rotation braking [70]. When combined with other DECAF warnings, these signals can contribute to a more robust real-time disruption warning system. Additionally, these warnings can provide input to a control algorithm which, for example, can apply actuators to maintain $\beta_{N}$ at a stable value [71].

\section{Cross-device application of machine learning assisted stability calculations}

Future high-powered fusion devices, such as ITER, will necessarily be operated in a much more disruption averse manner than present devices. If machine learning algorithms for disruption avoidance are to help, they must demonstrate that they can be trained on one device and reliably operated on another. Several efforts are underway to determine if this approach is feasible, including for example cross-machine comparisons of disruption 


\section{Physics-guided ML approaches for ideal stability forecasting}

forecasting between the DIII-D and JET tokamaks [20]. Here, our goal is to attempt to use the model trained on NSTX data and apply it to the most similar other device, the spherical tokamak MAST, to discover the advantages and limitations of such an approach. Naturally, the same process as was used here to train a neural network for determination of the no-wall beta limit, or a RFR for emulation of the DCON calculation of $\delta W_{n o-w a l l}^{n=1}$ can be repeated on the database of MAST equilibria, and it is our intention to do so in the future. However, in order to properly repeat this process, first high quality equilibrium reconstructions using kinetic profiles and motional Stark effect constraints on the q profile for the MAST database are required as the basis of the DCON calculations or as input to the machine learning algorithms. As these reconstructions are now being generated in present research, we can start with the available magnetics-only equilibrium reconstructions for some examples of cross-machine application. Although this is mostly future work, we will here briefly present some initial examples which show the promise of the approach.

First, we will utilize the NSTX-trained $\beta_{N}$ formula on two discharges (25109 and 25112) in MAST, for which the no-wall beta limit was experimentally probed by active MHD spectroscopy [72, 73] and calculated with the MISHKA-1 code [74].

Figure 11: $\beta_{N}$ vs. time with the neural network no-wall limit trained on NSTX data, for MAST discharges (a) 25109 and (b) 25112. The grey area indicates the uncertainty on the no-wall limit estimation, as per 9. 


\section{Physics-guided ML approaches for ideal stability forecasting}

Active MHD spectroscopy is an established experimental diagnostic technique used to measure MHD mode stability when the plasma is stable by measuring the resonant field amplification (RFA) of a travelling toroidal mode number $n=1$ applied tracer field. Experimental evidence to date has shown that increasing amplitude generally indicates decreasing mode stability and a sharply increasing amplitude can indicate the approach to the no-wall limit. By using magnetics-only equilibria and the NSTX derived no-wall limit formula from 8 without any changes for MAST, we can see in Fig. 11 that the predicted $\beta_{N, n o-w a l l}^{n=1}$ is somewhat larger than the RFA asymptote, but very close to the MISHKA-1 predictions.

It is worth noting that the operating point of these MAST discharges is just at the edge, or even above, the domain of applicability of the neural network results, with $l_{i}>$ 0.8 and $\mathrm{A}$ up to 1.6 at high $\beta_{N}$. Therefore, the NSTX-trained formula applied to MAST has been influenced by the physics guidance outside the training region. This helped to improve the MAST no-wall limit predictions, compared to the previous calculations, as otherwise they would have been even larger. As was stated, this analysis needs to be repeated with accurate kinetic equilibrium reconstructions and DCON calculations of $\beta_{N, n o-w a l l}^{n=1}$. This example also demonstrates the utility of using multiple machines to validate and refine a machine learning assisted physics model, as doing so can expand the domain of applicability. This can be tried by training the neural network with just a glimpse[20] of MAST data, since this might be the necessary path for future devices such as ITER where a small amount of initial data might be used to update previously trained algorithms, or by combining the full databases of both machines.

The second example of cross-machine application from an NSTX-trained machine learning approach to a MAST discharge is to attempt to emulate DCON calculations of $\delta W_{n o-w a l l}^{n=1}$. A very high beta MAST discharge, 7090 [75], was selected for this purpose. Figure $12(\mathrm{a})$ shows that $\beta_{N}$ peaks in this discharge at $\sim 5.5$, and the magnetics-only equilibrium is used as an input to DCON to calculate $-\delta W_{n o-\text { wall }}^{n=1}$ in Fig. $12(\mathrm{~b})$. The RFR trained on NSTX data (with kinetic equilibrium reconstructions) was then used to emulate $-\delta W_{n o-\text { wall }}^{n=1}$.

The trend is similar, although interestingly the DCON calculations show the no-wall limit (zero crossing) at around 5.2 while $\beta_{N}$ is increasing in the shot, and at 4.3 when it is decreasing, whereas the random forest results are more consistent, both times crossing at about $\beta_{N} \sim$ 4.6. The magnitude is also different, although the DCON results of $\delta W_{n o-w a l l}^{n=1}>6$ are unusually large based on the NSTX experience (see Fig. 3). Overall, $-\delta W_{n o-w a l l}^{n=1}$ predictions for this MAST discharge, based on a random forest trained on the NSTX device, seem quite reasonable and may be closer in magnitude when compared to potentially new DCON results from kinetic equilibria for MAST. This remains to be seen, but the results are encouraging. Of course, when the random forest predictions are compared to new MAST DCON runs, differences will surely be found as well, which will be informative and in future work may lead to a better predictor. 
1

2

3

4

5

6

7

8

Physics-guided ML approaches for ideal stability forecasting
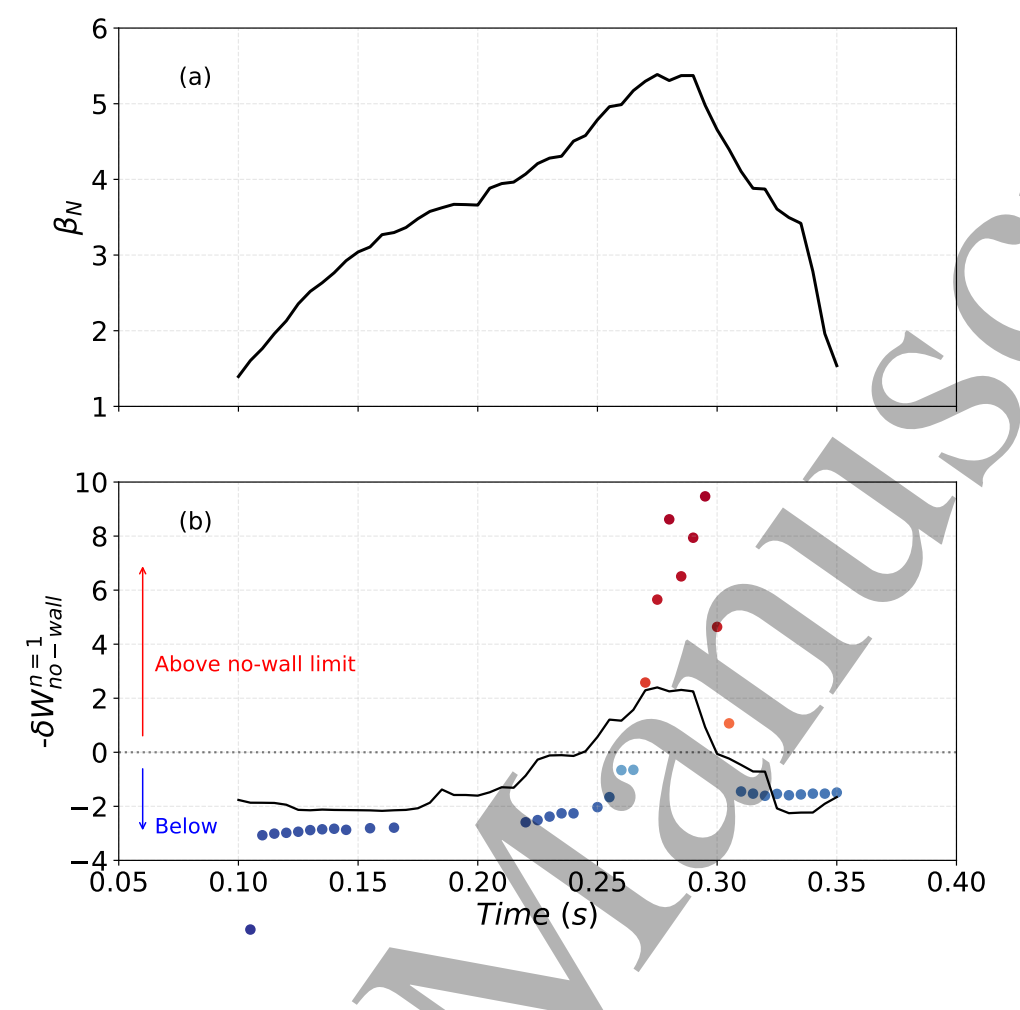

Figure 12: (a) $\beta_{N}$ vs. time and (b) $-\delta W_{n o-w a l l}^{n=1}$ calculations from DCON (colored points), and from the random forest algorithm trained on NSTX data (black line) for MAST discharge 7090 .

\section{Discussion and conclusions}

The reduced kinetic stability model is one of the core modules inside the DECAF framework and has been widely used to reproduce marginal stability points in NSTX. A novel approach combining prior physics knowledge and machine learning algorithms was explored to improve the underlying ideal stability calculations of the no-wall limit. Interpretable machine learning approaches, such as the random forest regression, can give an insight into the physics behind DCON calculations and provide the importance that each plasma quantity has in the determination of $\beta_{N, n o-\text { wall }}$ and $\delta W_{n o-w a l l}$. An improved closed-form equation of the no-wall limit has been derived by combining random forest weights with neural network defined decision boundaries in the $\beta_{N}$ versus $l_{i}, A$ and $p_{0} /\langle p\rangle$ operating spaces. This new formulation outperforms the previously defined equation, leading to a significant reduction of missed instabilities (false negatives) and consistently replicating DCON calculations in simulated real-time analyses.

There are various elements in this approach that may be improved. First of all, the current DECAF model incorporates modifications to ideal stability by kinetic effects 


\section{Physics-guided ML approaches for ideal stability forecasting}

that are left unchanged in the present work and that will be subject of further machine learning assisted calculations. Secondly, the usage of neural networks has revealed a caveat of many machine learning algorithms, which is the extrapolation outside of the training region. Here we have used ideal MHD theory as physics guidance for the neural network. A first cross-device application on a similar tokamak, MAST, is so far quite promising, especially since the neural network has not seen any MAST data. There is still room for improvement as soon as high quality equilibrium reconstructions and DCON runs are available for MAST, though.

In conclusion, a first attempt to include machine learning tools inside the DECAF framework has proved that physics knowledge and artificial intelligence can cooperate in order to build robust real-time disruption avoidance systems for future relevant fusion devices.

Work in the near future will cover a study of the kinetic effects and the possible procedures to improve the $\delta W_{K}$ term, which will provide a machine learning assisted alternative to the entire kinetic stability model. Moreover, domain adaptation is a necessary line of research in order to refine understanding of how kinetic RWM stability scales across different machines.

\section{Acknowledgments}

The authors acknowledge the help of M.D. Boyer, S.P. Gerhardt and R.J. Akers, and are also grateful to A. Abbas for the helpful discussions about customized objective functions.

This research was supported by EPSRC grants EP/R025290/1 and EP/P02243X/1 (University College London), and by U.S. Department of Energy under contract $D E$ SC0018623 (Columbia University).

[1] Berkery J W, Sabbaghy S A, Bell R E, Gerhardt S P and LeBlanc B P 2017 Phys. Plasmas 24 056103 URL https://doi.org/10.1063/1.4977464

[2] Kaye S M et al 2019 Nucl. Fusion 59112007 URL https://doi .org/10.1088/1741-4326/ab023a

[3] Strait E J et al 2019 Nucl. Fusion 59112012 URL https://doi .org/10.1088/1741-4326/ab15de

[4] Hu B and Betti/R 2004 Phys. Rev. Lett 93105002 URL https://doi.org/10.1103/PhysRevLett. 93.105002

[5] Berkery J W, Sabbagh S A, Betti R, Hu B, Bell R E, Gerhardt S P, Manickam J and Tritz K 2010 Phys. Rev. Lett 104035003 URL https://doi.org/10.1103/PhysRevLett.104.035003

[6] Sabbagh S A, Berkery J W, Bell R E, Bialek J M, Gerhardt S P, Menard J E, Betti R, Gates D A, Hu B, Katsuro-Hopkins O N, LeBlanc B P, Levinton F M, Manickam J, Tritz K and Yuh H 2010 Nucl. Fusion 50 025020 URL https://doi.org/10.1088/0029-5515/50/2/025020

[7] Berkery J W, Sabbagh S A, Bell R E, Gerhardt S P, LeBlanc B P and Menard J E 2015 Nucl. Fusion 55123007 URL https://doi.org/10.1088/0029-5515/55/12/123007

[8] Glasser A H 2016 Phys. Plasmas 23 072505 URL https://doi.org/10.1063/1.4958328

[9] Berkery J W, Sabbagh S A, Reimerdes H, Betti R, Hu B, Bell R E, Gerhardt S P, Manickam J and Podestà M 2010 Phys. Plasmas 17082504 URL https://doi.org/10.1063/1.3474925

[10] Glasser A S, Kolemen E and Glasser A H 2018 Phys. Plasmas 25032507 URL https://doi.org/ 10.1063/1.5007042 
[11] Hernandez J V, Vannucci A, Tajima T, Lin Z, Horton W and McCool S C 1996 Nucl. Fusion 36 1009 URL https://doi.org/10.1088/0029-5515/36/8/I05

[12] Wroblewski D, Jahns G L and Leuer J A 1997 Nucl. Fusion 37725 URL https://doi.org/10. 1088/0029-5515/37/6/I02

[13] Vannucci A, Oliveira K A and Tajima T 1999 Nucl. Fusion 39255 URL https://doi.org/10. 1088/0029-5515/39/2/308

[14] Pautasso G, Tichmann C, Egorov S, Zehetbauer T, Gruber O, Maraschek M, Mast K-F, Mertens V, Perchermeier I, Raupp G, Treutterer W, Windsor C G and ASDEX Upgrade Team 2002 Nucl. Fusion 42100 URL https://doi.org/10.1088/0029-5515/42/1/314

[15] Cannas B, Fanni A, Marongiu E and Sonato P 2004 Nucl. Fusion 4468 URL https://doi.org/ $10.1088 / 0029-5515 / 44 / 1 / 008$

[16] Windsor C G, Pautasso G, Tichmann C, Buttery R J, Hender T C, JET EFDA Contributors and the ASDEX Upgrade Team 2005 Nucl. Fusion 45337 URL https://doi.org/10.1088/ 0029-5515/45/5/004

[17] Yoshino R 2005 Nucl. Fusion 451232 URL https://doi.org/10.1088/0029-5515/45/11/003

[18] Ferreira D R, Carvalho P J, Fernades H and JET Contributors 2018 Fusion Science and Tech. 74 47 URL https://doi.org/10.1080/15361055.2017.1390386

[19] Zheng W, Hu F R, Zhang M, Chen Z Y, Zhao X Q, Wang X L, Shi P, Zhang X L, Zhang X Q, Zhou Y N, Wei Y N, Pan Y and J-TEXT Team 2018 Nucl. Fusion 58056016 URL https://doi.org/10.1088/1741-4326/aáad17

[20] Kates-Harbeck J, Svyatkovskiy A and Tang W 2019 Nature 568526 URL https://doi.org/10. 1038/s41586-019-1116-4

[21] Citrin J, Breton S, Felici F, Imbeaux F, Aniel T, Artaud J F, Baiocchi B, Bourdelle C, Camenen Y and Garcia J 2015 Nucl. Fusion 55092001 URL https://doi.org/10.1088/0029-5515/55/ 9/092001

[22] Meneghini O, Smith S P, Snyder P B, Staebler G,M, Candy J, Belli E, Lao L, Kostuk M, Luce T, Luda T, Park J M and Poli F 2017 Nucl. Fusion 57086034 URL https://doi.org/10.1088/ 1741-4326/aa7776

[23] Boyer M D, Kaye S M and Erickson K 2019 Nucl. Fusion 59056008 URL https://doi.org/10. 1088/1741-4326/ab0762

[24] Breiman L 2001 Machine Learning 455 URL https://doi.org/10.1023/A:1010933404324

[25] Rea C, Granetz R S, Montes K J, Tinguely R A, Eidietis N, Hanson J M and Sammuli B 2018 Plasma Phys. Control. Fusion 60084004 URL https://doi.org/10.1088/1361-6587/aac7fe

[26] Rea C and Granetz R S 2018 Fusion Science and Tech. 7489 URL https://doi.org/10.1080/ 15361055.2017 .1407206

[27] Rea C, Montes K J, Erickson K G, Granetz R S and Tinguely R A 2019 Nucl. Fusion 59096016 URL https://doi.org/10.1088/1741-4326/ab28bf

[28] Montes K J, Rea C, Granetz R S, Tinguely R A, Eidietis N, Meneghini O M, Chen D L, Shen B, Xiao B J, Erickson K and Boyer M D 2019 Nucl. Fusion 59096015 URL https: //doi.org/10.1088/1741-4326/ab1df4

[29] Tinguely R A, Montes K J, Rea C, Sweeney R and Granetz R S 2019 Plasma Phys. Control. Fusion 61095009 URL https://doi.org/10.1088/1361-6587/ab32fc

[30] Hender T C et al 2007 Nucl. Fusion 47 S128 URL https://doi.org/10.1088/0029-5515/47/6/ $\mathrm{S} 03$

[31] Boozer A H 2012 Phys. Plasmas 2012058101 URL https://doi.org/10.1063/1.3703327

[32] Eidietis N W, Gerhardt S P, Granetz R S, Kawano Y, Lehnen M, Lister J B, Pautasso G, Riccardo V, Tanna R L, Thornton A J and The ITPA Disruption Database Participants 2015 Nucl. Fusion 55063030 URL 10.1088/0029-5515/55/6/063030

[33] Aydemir A Y, Lee H H, Lee S G, Seol J, Park B H and In Y K 2016 Nucl. Fusion 56054001 URL 10.1088/0029-5515/56/5/054001

[34] Hollmann E M, Aleynikov P B, Fülöp T, Humphreys D A, Izzo V A, Lehnen M, Lukash V E, 
Physics-guided ML approaches for ideal stability forecasting

Papp G, Pautasso G, Saint-Laurent F and Snipes J A 2015 Phys. Plasmas 22021802 URL 10.1063/1.4901251

[35] Baylor L R, Barbier C C, Carmichael J R, Combs S K, Ericson M N, Bull Ezel N D, Fisher P W, Lyttle M S, Meitner S J, Rasmussen D A, Smith S F, Wilgen J B, Maruyama S and Kiss G 2015 Fusion Science and Tech. 68211 URL https://doi.org/10.13182/FST14-926

[36] de Vries P C, Pautasso G, Humphreys D, Lehnen M, Maruyama S, Snipes J A, Vergara A and Zabeo L 2016 Fusion Science and Tech. 69471 URL https://doi.org/10.13182/FST15-176

[37] Geelen P, Felici F, Merle A and Sauter O 2015 Plasma Phys. Control. Fusion 57125008 URL https://doi.org/10.1088/0741-3335/57/12/125008

[38] Blanken T C, Felici F, Galberti C, Vu N M T, Kong M, Sauter O, de Baar M R, The EUROfusion MST1 Team and The TCV Team 2019 Nucl. Fusion 59026017 URL https: //doi.org/10.1088/1741-4326/aaf451

[39] Pau A, Cannas B, Fanni A, Sias G, Baruzzo M, Murari A, Pautasso G, Tsalas M, JET Contributors, The ASDEX Upgrade Team and The EUROfusion MST1 Team 2017 Fusion Eng. and Design 125139 URL https://doi.org/10.1016/j.fusengdes.2017.10.003

[40] Bo W, Granetz R, Bingjia X, Jiangang L, Fei Y, Junjun L and Dalong C 2016 Plasma Science and Tech. 181162 URL https://doi.org/10.1088/1009-0630/18/12/04

[41] Murari A, Vega J, Rattá G A, Vagliasindi G, Johnson M F, Hong S H and JET-EFDA Contributors 2009 Nucl. Fusion 49 055028 URL https://doi.org/10.1088/0029-5515/49/5/055028

[42] Vega J, Dormido-Canto S, López J M, Murări A, Ramírez J M, Moreno R, Ruiz M, Alves D, Felton R and JET-EFDA Contributors 2013 Fusion Eng. and Design 881228 URL https: //doi.org/10.1016/j.fusengdes.2013.03.003

[43] Moreno R, Vega J, Dormido-Canto S, Pereira A, Murari A and JET Contributors 2016 Fusion Science and Tech. 69485 URL https://doi.org/10.13182/FST15-167

[44] Yokoyama T, Miyoshi Y, Hiwatari R, Isayama A, Matsunaga G, Oyama N, Igarashi Y, Okada M and Ogawa Y 2019 Fusion Eng. and Design 14067 URL https://doi.org/10.1016/j. fusengdes.2019.01.128

[45] Pau A, Fanni A, Carcangiu S, Cannas B, Sias G, Murari A, Rimini F and the JET Contributors 2019 Nucl. Fusion 59106017 URL https://doi.org/10.1088/1741-4326/ab2ea9

[46] Cranmer M D, Rui X, Battaglia P and Ho S 2019 ArXiv (Preprint https://arxiv.org/abs/ 1909.05862v2)

[47] Bondeson A and Ward D J 1994 Phys. Rev. Lett. 72 2709 URL https://doi.org/10.1103/ PhysRevLett.72.2709

[48] Sabbagh S A, Bell R E, Bell M G, Bialek J M, Glasser A H, LeBlanc B P, Menard J E, Paoletti F, Stutman D, Fredrickson E, Garofalo A M, Gates D A, Kaye S M, Lao L L, Maingi R, Mueller D, Navratil G, Ono M, Peng M, Synakowski E, Zhu W and NSTX Research Team 2002 Phys. Plasmas 92085 URL https://doi.org/10.1063/1.1468230

[49] Sabbagh S A, Bell R E, Menard J E, Gates D A, Sontag A C, Bialek J M, LeBlanc B P, Levinton F M, Tritz K and Yuh H/2006 Phys. Rev. Lett. 97045004 URL https://doi.org/10.1103/ PhysRevLett.97.045004

[50] Chu M-S and Okabayashi M 2010 Plasma Phys. Control. Fusion 52123001 URL https: //doi.org/10.1088/0741-3335/52/12/123001

[51] Strait E J, Taylor T S, Turnbull A D, Ferron J R, Lao L L, Rice B, Sauter O, Thompson S J and Wróblewski D 1995 Phys. Rev. Lett. 742483 URL https://doi.org/10.1103/PhysRevLett. 74.2483

[52] Sabbagh S A, Sontag A C, Bialek J M, Gates D A, Glasser A H, Menard J E, Zhu W, Bell M G, Bell R E, Bondeson A, Bush C E, Callen J D, Chu M S, Hegna C C, Kaye S M, Lao L L, LéBlanc B P, Liu Y Q, Maingi R, Mueller D, Shaing K C, Stutman D, Tritz K and Zhang C 2006 Nucl. Fusion 46635 URL https://doi.org/10.1088/0029-5515/46/5/014

[53] Gerhardt S P, Menard J E, Sabbagh S A and Scotti F 2012 Nucl. Fusion 52063005 URL https://doi.org/10.1088/0029-5515/52/6/063005 
Physics-guided ML approaches for ideal stability forecasting

[54] Gerhardt S P 2013 Nucl. Fusion 53 023005 URL https://doi.org/10.1088/0029-5515/53/2/ 023005

[55] Hu B, Betti R and Manickam J 2005 Phys. Plasmas 12057301 URL https://doi.org/10.1063/ 1.1873852

[56] Berkery J W, Betti R, Sabbagh S A, Guazzotto L and Manickam J 2014 Phys. Plasmas 21112505 URL https://doi.org/10.1063/1.4901568

[57] Menard J E, Jardin S C, Kaye S M, Kessel C E and Manickam J 1997 Nucl. Fusion 37595 URL https://doi.org/10.1088/0029-5515/37/5/I03

[58] Turnbull A D, Taylor T S, Chu M S, Miller R L and Lin-Liu Y R 1998 Nucl. Fusion 381467 URL https://doi.org/10.1088/0029-5515/38/10/305

[59] Huysmans G T A, Hender T C, Alper B, Baranov Y F, Borba D, Conway G D, Cottrell G A, Gormezano C, Helander P, Kwon O J, Nave M F F, Sips A C C, Söldner F X, Strait E J, Zwingmann W P and JET Team 1999 Nucl. Fusion 391489 URL https://doi.org/10.1088/ 0029-5515/39/11/301

[60] Troyon F, Gruber R, Saurenmann H, Semenzato S and Succi S 1984 Plasma Phys. Control. Fusion 26209 URL https://doi.org/10.1088/0741-3335/26/1A/319

[61] Menard J E, Bell M G, Bell R E, Gates D A, Kaye S M, LeBlanc B P, Maingi R, Sabbagh S A, Soukhanovskii V, Stutman D and NSTX National Research Team 2004 Phys. Plasmas 11639 URL https://doi.org/10.1063/1.1640623

[62] Karpatne A, Atluri G, Faghmous J H, Steinbach M, Banerjee A, Ganguly A, Shekhar S, Samatova N and Kumar V 2017 IEEE Trans. Knowledge Data Eng. 292318 URL https: //doi.org/10.1109/TKDE.2017.2720168

[63] Karpatne A, Watkins W, Read J and Kumar V 2017 ArXiv (Preprint https://arxiv.org/abs/ $1710.11431 \mathrm{v} 2)$

[64] Raissi M, Perdikaris P and Karniadakis G E 2019 Journal of Comp. Phys. 378 686 URL https://doi.org/10.1016/j.jcp.2018.10.045

[65] Dormann C F, Elith J, Bacher S, Buchmann C, Gudrun C, Carré G, García Marquéz J R, Gruber B, Lafourcade B, Leitão P J, Münkemüller T, McClean C, Osborne P E, Reineking B, Schröder B, Skidmore A K, Zurell D and Lautenbach S 2013 Ecography 3627 URL https://doi.org/10.1111/j.1600-0587.2012.07348.x

[66] Migut M A, Worring M and Veenman C J 2015 Data Min. Knowl. Disc. 29273 URL https: //doi.org/10.1007/s10618-013-0342-x

[67] Karimi H, Derr T and Tang J 2019 ArXiv (Preprint https://arxiv.org/pdf/1912.11460v2.pdf)

[68] Chollet F 2018 Deep Learning with Python (Manning Publications) URL https://www.manning. com/books/deep-learning-with-python

[69] Goodfellow I, Bengio Y and Courville A 2016 Deep Learning (MIT Press) URL https://www. deeplearningbook.org/

[70] Piovesan P, Igochine V, Turco F, Ryan D A, Cianciosa M R, Liu Y Q, Marrelli L, Terranova D, Wilcox R S, Wingen A, Angioni C, Bock A, Chrystal C, Classen I, Dunne M, Ferraro N M, Fischer R, Gude A, Holeomb C T, Lebschy A, Luce T C, Maraschek M, McDermott R, Odstril T, Paz-Soldan C, Reich M, Sertoli M, Suttrop W, Taylor N Z, Weiland M, Willensdorfer M, The ASDEX Upgrade Team, The DIII-D Team and The EUROfusion MST1 Team 2017 Plasma Phys. Control. Fusion 59 014027 URL https://doi.org/10.1088/0741-3335/59/1/014027

[71] Boyer M D, Andre R, Gates D A, Gerhardt S P, Goumiri I R and Menard J E 2015 Nucl. Fusion 55053033 URL https://doi.org/10.1088/0029-5515/55/5/053033

[72] Chapman I T, Cooper W A, Graves J P, Gryaznevich M P, Hastie R J, Hender T C, Howell D F, Hua M-D, Huysmans G T A, Keeling D L, Liu Y Q, Meyer H F, Michael C A, Pinches S D, Saarelma S, Sabbagh S A and the MAST Team 2011 Nucl. Fusion 51073040 URL https://doi.org/10.1088/0029-5515/51/7/073040

[73] Chapman I T, Gryaznevich M P, Howell D F, Liu Y Q and the MAST Team 2011 Plasma Phys. Control. Fusion 53 065022 URL https://doi.org/10.1088/0741-3335/53/6/065022 
Physics-guided ML approaches for ideal stability forecasting

[74] Mikhailovskii A B, Huysmans G T A, Sharapov S E and Kerner W 1997 Plasma Phys. Rep. 23 844

[75] Hole M J, Akers R J, Appel L C, Buttery R J, Brickley C, Conway N J, Gryaznevich M, Hender T C, Kwon O J, Valovic M, Medvedev S, Patel A, Saarelma S, Taylor D, Wilson H R and the MAST Team 2005 Plasma Phys. Control. Fusion 47581 URL https://doi.org/10.1088/ 0741-3335/47/4/002

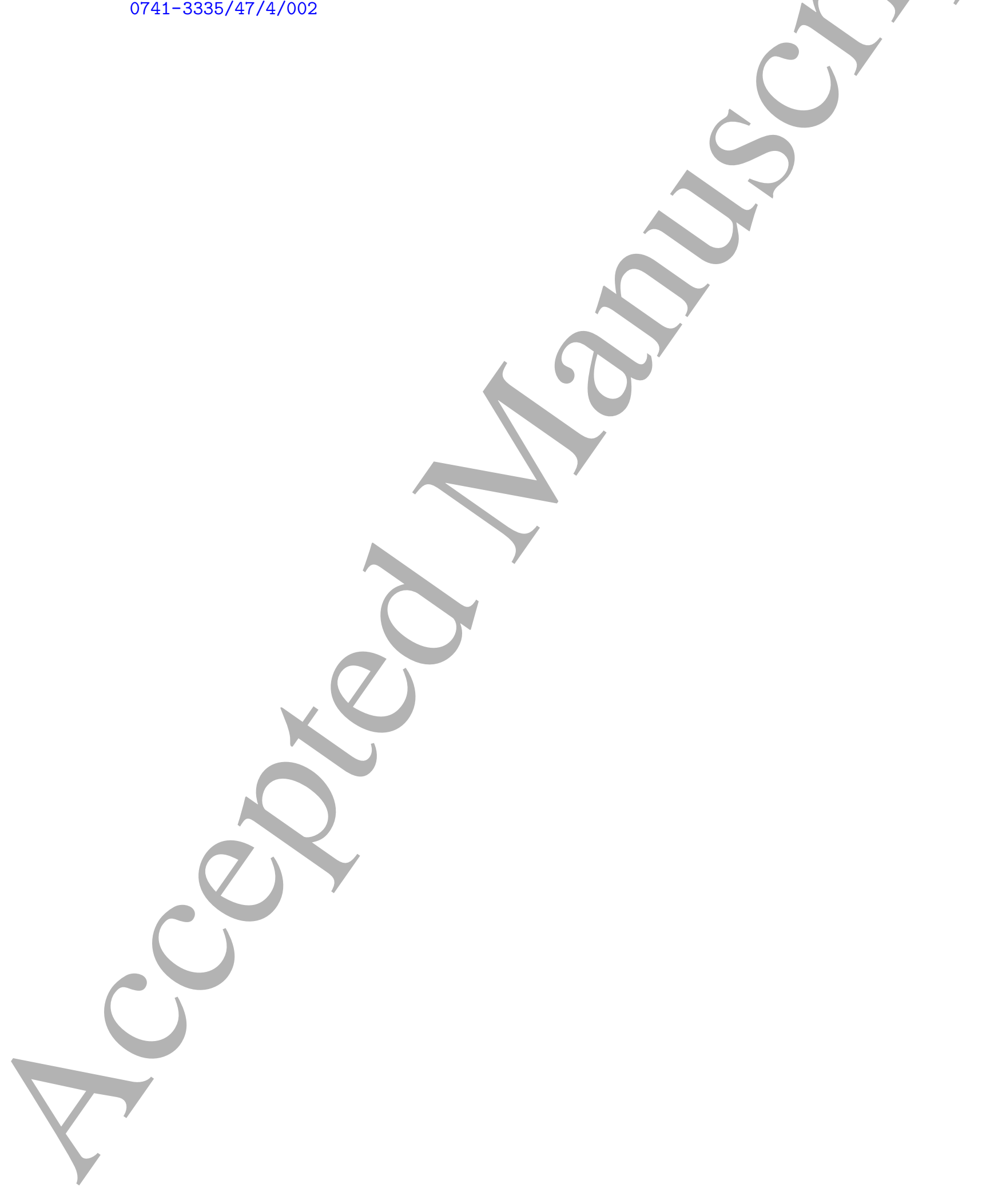

